\title{
Slit-Robo signaling induces malignant transformation through Hakai-mediated E-cadherin degradation during colorectal epithelial cell carcinogenesis
}

Wei-Jie Zhou ${ }^{1, *}$, Zhen H Geng ${ }^{2, *}$, Shan Chi ${ }^{1, *}$, Wenli Zhang ${ }^{3, *}$, Xiao-Feng Niu ${ }^{1, *}$, Shu-Jue Lan ${ }^{1, *}$, Li Ma ${ }^{2}$, Xuesong Yang ${ }^{4}$, Li-Jing Wang $^{5}$, Yan-Qing Ding ${ }^{3}$, Jian-Guo Geng $^{2,5}$

${ }^{I}$ Laboratory of Molecular Cell Biology, Institute of Biochemistry and Cell Biology, Shanghai Institutes for Biological Sciences, Chinese Academy of Sciences, 320 Yue-Yang Road, Shanghai 200031, China; ${ }^{2}$ Department of Biologic and Materials Sciences, University of Michigan School of Dentistry, Ann Arbor, MI 48109, USA, ${ }^{3}$ Department of Pathology, Nanfang Hospital and School of Basic Medical Sciences, Southern Medical University, Guangzhou, Guangdong 510515, China; ${ }^{4}$ Key Laboratory for Regenerative Medicine of the Ministry of Education, Medical College, Jinan University, Guangzhou, Guangdong 510632, China; ${ }^{5}$ Vascular Biology Research Institute, Guangdong Pharmaceutical University, Guangzhou, Guangdong 510006, China

The Slit family of guidance cues binds to Roundabout (Robo) receptors and modulates cell migration. We report here that ectopic expression of Slit2 and Robo1 or recombinant Slit2 treatment of Robo1-expressing colorectal epithelial carcinoma cells recruited an ubiquitin ligase Hakai for E-cadherin (E-cad) ubiquitination and lysosomal degradation, epithelial-mesenchymal transition (EMT), and tumor growth and liver metastasis, which were rescued by knockdown of Hakai. In contrast, knockdown of endogenous Robo1 or specific blockade of Slit2 binding to Robo1 prevented E-cad degradation and reversed EMT, resulting in diminished tumor growth and liver metastasis. Ectopic expression of Robo1 also triggered a malignant transformation in Slit2-positive human embryonic kidney 293 cells. Importantly, the expression of Slit2 and Robo1 was significantly associated with an increased metastatic risk and poorer overall survival in colorectal carcinoma patients. We conclude that engagement of Robo1 by Slit2 induces malignant transformation through Hakai-mediated E-cad ubiquitination and lysosomal degradation during colorectal epithelial cell carcinogenesis.

Keywords: colorectal carcinogenesis; E-cadherin; Hakai; Robo; Slit

Cell Research (2011) 21:609-626. doi:10.1038/cr.2011.17; published online 1 February 2011

\section{Introduction}

The Slit family of guidance cues interacts with the Roundabout (Robo) family of transmembrane receptors in a wide variety of physiological processes requiring cell migration [1-4]. Slit-Robo signaling regulates the repulsion or attraction of projecting axons, and migrating neurons during development of the nervous system [5, 6]. Slit 2 secreted by vascular endothelial cells binds to

\footnotetext{
*These six authors contributed equally to this work.

Correspondence: Jian-Guo Geng ${ }^{\mathrm{a}}$, Yan-Qing Ding ${ }^{\mathrm{b}}$

a'E-mail: genglab@gmail.com

be-mail: dyqsmu@sina.com

Received 9 September 2010; revised 19 September 2010; accepted 21 September 2010; published online 1 February 2011
}

Robo1 on leukocytes and acts as an endogenous inhibitor of leukocyte chemotaxis [7-12]. Additionally, Slit2 mediates directional migration of malignant cells [13-15]. We and others have previously reported that Slit proteins secreted by solid tumors binds to Robol expressed on vascular and lymphatic endothelial cells to stimulate angiogenesis and lymphangiogenesis [16-22]. Although such paracrine pathways involving Slit-Robo interactions have been extensively studied, the biological significance and molecular mechanisms of autocrine Slit-Robo signaling are largely unexplored.

E-cadherin (E-cad) functions as a cell adhesion molecule in adherens junction [23-26]. The cytoplasmic region of E-cad directly interacts with $\beta$-catenin ( $\beta$-cat), which in turn interacts with $\alpha$-catenin $(\alpha$-cat) and other molecules to link it to the actin cytoskeleton [27]. Loss 
of E-cad leads to cell-cell dissociation and acquisition of a migratory phenotype during development, tissue remodeling or carcinogenesis [28-35]. In addition to E-cad, other molecular constituents of the adherens junction have important roles in maintaining cell-cell adhesion within the epithelium and preventing tumor invasiveness during carcinogenesis. Among them, $\alpha$-cat is essential for coordinating actin dynamics and inversely linking cell adhesion with proliferation [36].

We have previously reported elevated expression of Slit2 in human colorectal carcinoma tissues and cell lines [16]. Using cDNA microarray, a significant upregulation of Robo1 was found in colorectal carcinoma tissues [37]. Overexpression of Robol is also detected in transforming growth factor (TGF)- $\beta$ and Wnt-induced intestinal tumors [38]. Taken together, these results suggest the possibility of an autocrine mechanism through which colorectal carcinoma cells secrete Slit2 for signaling through Robo1 expressed on these same cells. This study investigated the biological significance and the molecular mechanism of autocrine Slit-Robo signaling in recruitment of Hakai, ubiquitination and lysosomal degradation of E-cad, cumulating to induction of epithelial-mesenchymal transition (EMT) and malignant transformation during the pathogenesis of colorectal epithelial cell carcinoma. Various in vitro assays, in vivo animal models, and analysis of patient samples were used to evaluate the effects of Slit2-Robo1 interaction on carcinogenesis of colorectal epithelial cells.

\section{Results}

Expression of Slit2 and Robol in human colorectal carcinoma cells

We examined the expression of Slit2 and Robo1 in 10 human colorectal carcinoma cell lines using Slit2 and Robo1 monoclonal antibodys (mAbs) [16]. Elevated Slit2 secretion or Robo1 and Slit2 expression $(\sim 180 \mathrm{kDa}$ for Slit2 and $\sim 200 \mathrm{kDa}$ for Robo1) was seen in the supernatants and lysates of six cell lines: SW620, SW480, LS180, LS174T, Lovo and Caco2 cells (Figure 1A). However, neither Robo1 nor Slit2 secretion/expression was evident in the other four cell lines; SW48, HCT116, HT29 and DLD1 cells. Human embryonic kidney 293 (HEK293) cells stably overexpressing Slit2 or Robo1 [16] were used as positive controls. $\alpha$-Tubulin $(\sim 55 \mathrm{kDa})$ was used in parallel as a sample loading control. The protein expression profile for each cell line was consistent with its mRNA levels for Robo1-4 and Slit1-3, as determined by quantitative real-time reverse transcription-polymerase chain reaction (qRT-PCR; Supplementary information, Data S1 and Figure S1A). Robo4 mRNA was not detected in any of the cell lines, even though it was clearly present in human umbilical vein endothelial cells (data not shown). Notably, Slit2 secretion or Slit2 and Robo1 expression was higher in metastatic SW620 cells than SW480 cells isolated from primary tumor (Supplementary information, Figure S1B), suggesting that secreted Slit2 may bind to cell-surface Robo1 to initiate Slit2-Robo1 signaling in colorectal carcinoma cells.

Slit-Robo signaling triggers a fibroblast-like morphology

E-cad is required for normal epithelial cell morphology and formation of cell-cell adherens junctions in the epithelial monolayer [23-26]. We, thus, speculated whether Slit-Robo signaling may alter cell morphology through downregulation of E-cad and other epithelial cell markers. To test this hypothesis, we performed gain- or loss-of-function studies in HCT116 cells lacking detectable Slit2 and Robo1 expression and SW620 cells overexpressing endogenous Slit2 and Robo1 (Figure 1A). Compared with HCT116 cells stably transfected with plain vector (HCT116/V), HCT116 cells stably transfected with Robo1 (HCT116/Robo1), Slit2 (HCT116/Slit2) or Slit2 plus Robo1 (HCT116/Robo1/Slit2) exhibited increased expression of their respective transgene products (Figure 1B, left panel and data not shown). Compared with SW620 cells stably transfected with plain vector (SW620/V), stable knockdown of Robol RNA by short hairpin RNA (shRNA) technology in SW620 cells (SW620/shRobo1) resulted in decreased levels of Robol protein (Figure 1B, right panel). Notably, we previously generated $\mathrm{R} 5$, a mAb that recognizes the first immunoglobulin-like domain of human, rat and mouse Robo1, and neutralizes Slit2 binding [16]. Using isolated recombinant human Slit2 (hSlit2) and recombinant human Robo1-Fc (hRobo1-Fc; Supplementary information, Figure S2A), we found that R5, but not isotype-matched irrelevant mouse immunoglobulin $\mathrm{G}$ ( $\mathrm{mIgG})$, inhibited the binding of human Robo1-Fc to immobilized hSlit2 (Supplementary information, Data S1 and Figure S2B). These data confirmed that R5 acts as a neutralizing $\mathrm{mAb}$ to Robo1 that prevents Slit2 recognition. We extensively used hSlit2 to mimic autocrine Slit2-Robo1 signaling for stimulation of Robo1-expressing cells or R5 to block the binding of endogenous Slit2 to Robol in the experiments.

Surprisingly, HCT116/Robo1/Slit2 cells developed elongated and spindle-shaped morphology that resembled fibroblast-like mesenchymal cells, whereas no such change in morphology was observed in HCT116/V, HCT116/Robo1 or HCT116/Slit2 cells (Figure 1C, upper panel and data not shown). Following knockdown of Robo1, SW620/shRobo1 cells developed a round cobble- 


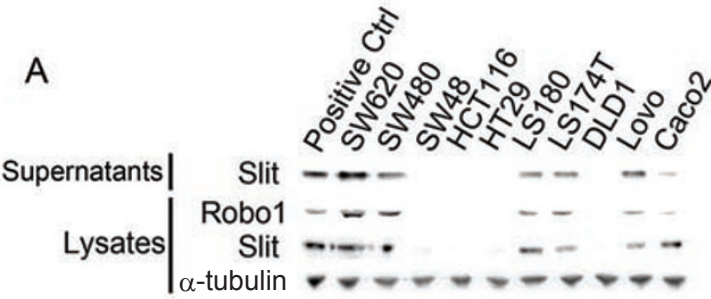

C

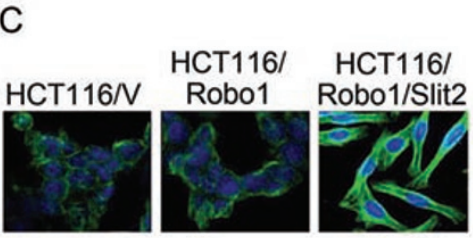

SW620N SW620/shRobo1
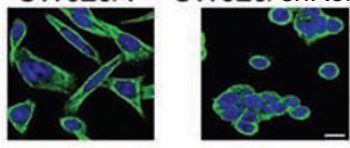

D
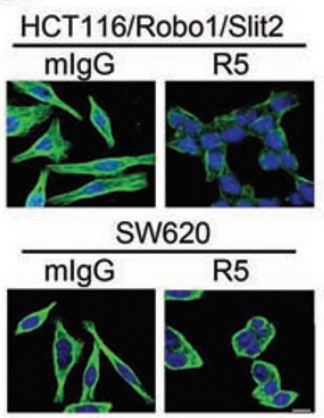

B

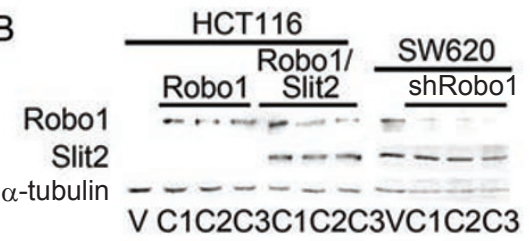

E

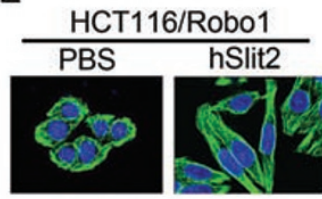

HCT116/Robo1+hSlit2 mlgG
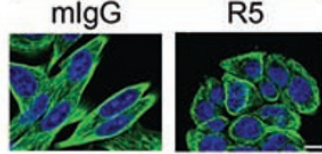

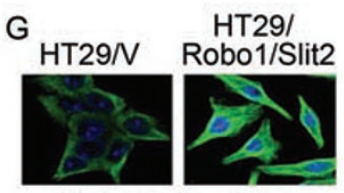

HT29/Robo1/Slit2 mlgG (e)

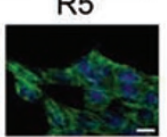

K

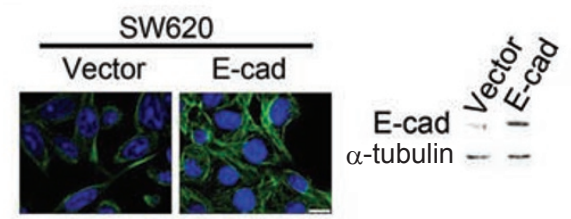

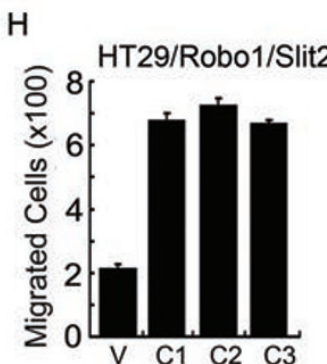

I HCT116/Robo1/Slit2

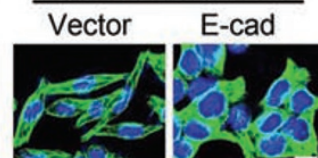

E-cad

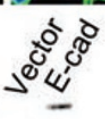
$\alpha$-tubulin - -

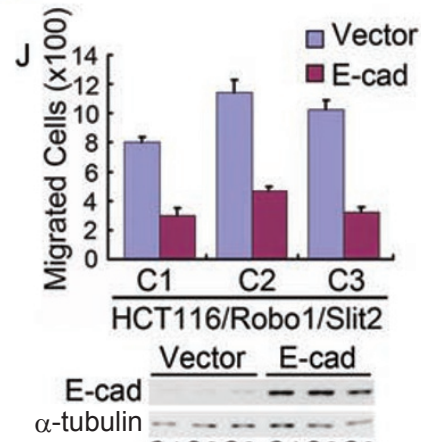

SW620 $\mathrm{C} 1 \mathrm{C} 2 \mathrm{C} 3 \mathrm{C}_{1} \mathrm{C}_{2} \mathrm{C} 3$

Figure 1 Slit-Robo signaling elicits fibroblast-like morphology. (A) Slit2 and Robo1 expression in human colorectal carcinoma cell lines detected by immunoblotting analysis with mAbs to Robo1, Slit2 and $\alpha$-tubulin. (B) HCT116 cells stably transfected with plain vector (HCT116/V) or plasmid encoding Robo1 (HCT116/Robo1) or Robo1 plus Slit2 (HCT116/Robo1/Slit2) and SW620 cells stably transfected with plain vector (SW620/V) or plasmid encoding shRNA for Robo1 knockdown (SW620/ shRobo1). Detergent lysates were immunoblotted with the Robo1, Slit2 and $\alpha$-tubulin mAbs. C1-3, single cell clones 1-3. (CF) Morphological features of HCT116/V, HCT116/Robo1 (C1), HCT116/Robo1/Slit2 (C1), SW620/V and SW620/shRobo1 (C1) cells in the absence and presence of $\mathrm{mlgG}, \mathrm{R} 5, \mathrm{PBS}$ and hSlit2 (C-E) or transfected with the plain vector or the plasmid of rat Robo1 (F). They were immunofluorescently stained for $\alpha$-tubulin (green) and nuclear DNAs with DAPI (blue). (G-L) Morphologic and migratory features of HT29/V and HT29/Robo1/Slit2 cells, HCT116/Robo1/Slit2 cells or SW620 cells transfected with the plain vector or the plasmid of E-cad in the absence and presence of mlgG or R5. Cell migratory activities were determined using the Boyden chamber assay as described previously [16]. Lysates were also immunoblotted for E-cad and $\alpha$-tubulin. Bars, $10 \mu \mathrm{m}$. Results are representative of at least three separate experiments (A-G, I and $\mathbf{K})$ or the mean \pm S.D. of three independent experiments $(\mathbf{H}, \mathbf{J}$ and $\mathbf{L})$. 


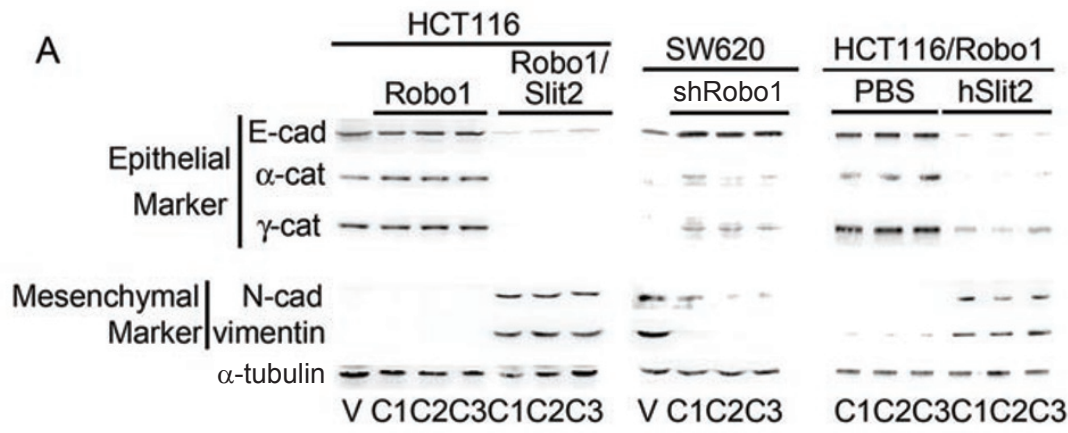

B
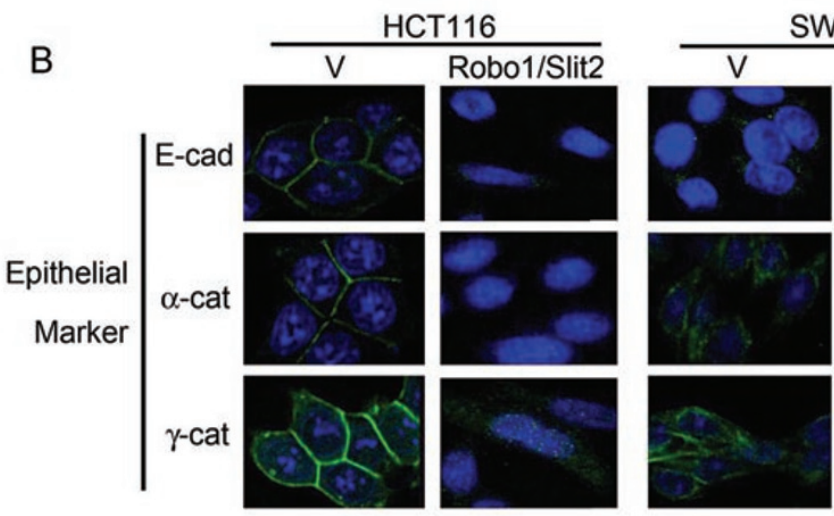

SW620
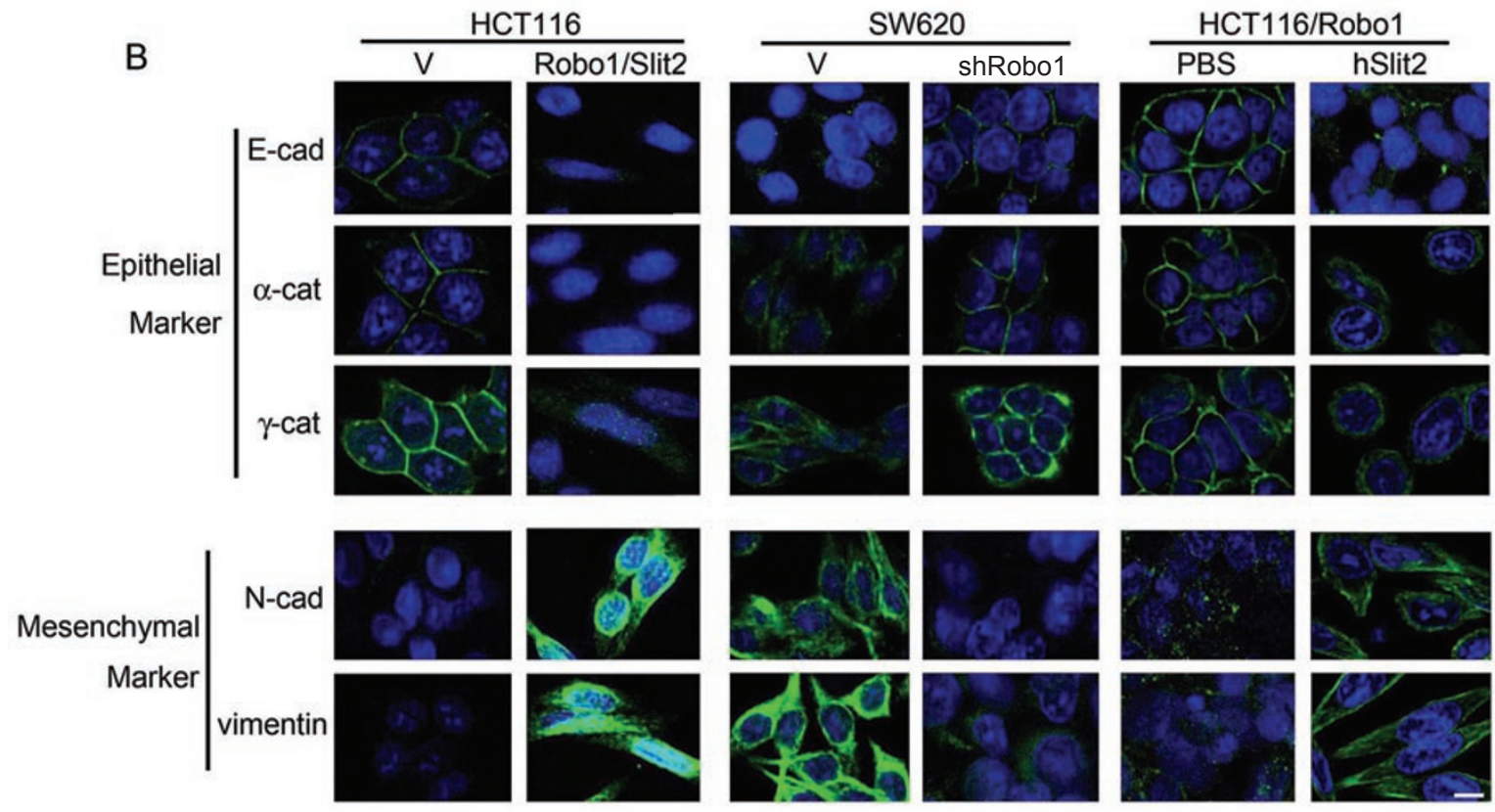

Figure 2 Slit-Robo signaling elicits mesenchmal markers. Expression of epithelial markers and mesenchymal markers in HCT116/V, HCT116/Robo1, HCT116/Robo1/Slit2, SW620/V and SW620/shRobo1 cells in the absence and presence of PBS and hSlit2. They were detected by immunoblotting (A) and immunofluorescent staining (B). HCT116/Robo1 (C1), HCT116/ Robo1/Slit2 (C1) and SW620/shRobo1 (C1) cells were used for immunofluorescent staining as above. Bars, $10 \mu \mathrm{m}$. Results are representative of at least three independent experiments.

stone shape similar in morphology to epithelial cells (Figure 1C, lower panel). Analogously, HCT116/Robo1/ Slit2 cells (Figure 1D, upper panel) and SW620 cells (Figure 1D, lower panel) acquired an epithelial cell-like phenotype when they were treated with R5, but not with mIgG. Incubation with hSlit2 switched HCT116/Robo1 cells from an epithelial-like morphology to a mesenchymal-like morphology (Figure 1E, upper panel), which was neutralized by R5, but not by $\mathrm{mIgG}$ (Figure 1E, lower panel), attesting to the functional significance of Slit2 in induction of fibroblast-like morphology for colorectal epithelial cells. Following transfection with the plasmid of rat Robo1 (rRobo1) or the plain vector (V), we found that only rRobo1-reintroduced SW620/shRobo1 cells back to a mesenchymal-like morphology (Figure $1 \mathrm{~F}$ ), thus eliminating the possibility of an off-target effect for the Robo1 shRNA plasmid used.

In support of our findings, we found that in analogous to HCT116 cells, HT29 cells lacked expression of Slit2 and Robol (Figure 1A). However, stable overexpression of Robo1 and Slit2 in HT29 (HT29/Robo1/Slit2) cells also induced a fibroblast-like morphology with long, slender processes compared with HT29 cells transfected with the plain vector $($ HT29/V), which was reversed by treatment with R5, but not with $\mathrm{mIgG}$ (Figure 1G). Compared with the plain vector, ectopic expression of E-cad (Figure 1I, lower panel and Figure 1K, right panel) switched the mesenchymal-like morphology back to the epithelial-like morphology in HCT116/Robo1/Slit2 cells (Figure 1I, upper panel) and SW620 cells (Figure 
$1 \mathrm{~K}$, left panel), further demonstrating the importance of Slit-Robo signaling-induced E-cad modulation in maintenance of epithelial-like features in the morphology of colorectal epithelial cells.

\section{Slit-Robo signaling induces EMT markers}

We next assessed whether Slit2-Robol signaling modulates cell morphology in a way indicative of EMT in colorectal carcinoma cells. Compared with HCT116/ V cells and HCT116/Robo1 cells, HCT116/Robo1/Slit2 cells displayed dramatically decreased expression of the epithelial markers E-cad $(\sim 120 \mathrm{kDa}), \alpha$-cat $(\sim 102 \mathrm{kDa})$ and $\gamma$-cat $(\sim 82 \mathrm{kDa})$, along with significantly increased expression of the mesenchymal markers N-cad $(\sim 120$ $\mathrm{kDa})$ and vimentin ( $\sim 58 \mathrm{kDa}$; Figure $2 \mathrm{~A}$ and $2 \mathrm{~B}$, left panels). In contrast, SW620/shRobol cells showed the opposite profile of these molecular markers, with evidence of augmented expression of E-cad, $\alpha$-cat and $\gamma$-cat and reduced the expression of $\mathrm{N}$-cad and vimentin, as compared with SW620/V cells (Figure 2A and 2B, middle panels). Furthermore, incubation of HCT116/Robo1 cells with hSlit2, but not phosphate-buffered saline (PBS), downregulated E-cad, $\alpha$-cat and $\gamma$-cat while upregulating $\mathrm{N}$-cad and vimentin (Figure 2A and 2B, right panels). On the basis of these findings, it is reasonable to surmise that Slit2-Robo1 signaling modulates the expression of E-cad and other EMT markers to reversibly control the transition between epithelial and mesenchymal phenotypes.

\section{Slit-Robo signaling enhances tumor growth and liver metastasis}

Given that Slit-Robo signaling induces EMT in colorectal carcinoma cells, we examined whether Slit-Robo signaling promotes cell transformation by increasing cell proliferation and migration, and thereby enhancing tumor growth and metastasis. Compared with HCT116/V cells, HCT116/Robo1/Slit2 cells grew faster (Figure 3A), migrated more rapidly (Figure $3 \mathrm{~B}$ ), and formed more colonies (Figure $3 \mathrm{C}$ ) in vitro. When inoculated subcutaneously into athymus nude mice, HCT116/Robo1/Slit2 cells grew faster (Figure 3D) and metastasized to the liver (Figure 3E) in vivo. In contrast, SW620/shRobo 1 cells grew more slowly (Figure 3F), migrated less rapidly (Figure $3 \mathrm{G}$ ) and formed fewer colonies (Figure 3H) in vitro compared with SW620/V cells. Mice inoculated subcutaneously with SW620/shRobol cells had dramatically reduced tumor volumes (Figure 3I) and no liver metastasis (Figure 3J). Treatment of HCT116/Robo1/ Slit2 and SW620 cells with R5, but not with $\mathrm{mIgG}$, also inhibited in vitro proliferation (Figure $3 \mathrm{~K}$ and $3 \mathrm{~L}$ ), migration (Figure $3 \mathrm{M}$ ) and colony formation (Figure $3 \mathrm{~N}$ ). In mice inoculated subcutaneously with HCT116/Robo1/
Slit2 cells or SW620 cells, R5, but not mIgG, attenuated in vivo tumor growth (Figure $3 \mathrm{O}$ and $3 \mathrm{P}$ ) and metastasis (Figure 3Q). Similarly, HT29/Robo1/Slit2 cells manifested enhanced migration compared with HT29/V cells (Figure 1H), which was inhibited by R5, but not by $\mathrm{mIgG}$ (data not shown). Overexpression of E-cad (Figure 1J, lower panel and Figure 1L, right panel) reduced the ability of HCT116/Robo1/Slit2 cells (Figure 1J, upper panel) and SW620 cells (Figure 1L, left panel) to migrate through the membranes of Boyden chamber. As the matter of fact, it was quite amazing to us to find that ectopic expression of Slit2 and Robo1 markedly accelerated HCT116 cell growth (Figure 3D, right panel), while knockdown of Robo1 almost abolished SW620 cell growth (Figure 3I, right panel) in the murine xenograft model. Taken together, these in vitro and in vivo results demonstrate that Slit-Robo signaling potently promotes tumor growth and metastasis by drastically increasing growth, migration and transformation of colorectal epithelial cells.

\section{Slit-Robo signaling recruits Hakai and ubiquitinates E-} cad for lysosomal localization

Hakai is an E3 ubiquitin ligase that is known to mediate E-cad ubiquitination and lysosomal degradation [31, 39]. We thus investigated whether Slit-Robo signaling might recruit Hakai to E-cad. Compared with HCT116/V and HCT116/Robo1 cells, ectopic expression of Slit2 and Robol enhanced the association of E-cad with Hakai in HCT116/Robo1/Slit2 cells, as detected by the anti-Hakai $\mathrm{Ab}$ from Santa Cruz Biotechnology (Figure 4A, left panel; $\sim 60 \mathrm{kDa}$ ). The E-cad-Hakai association in HCT116/ Robo1/Slit2 cells was inhibited by R5, but not by $\mathrm{mIgG}$ (Figure 4C, left panel). The E-cad-Hakai association in SW620 cells was also blocked by knockdown of Robo1 mRNA (Figure 4B, left panel) or treatment with R5 (Figure $4 \mathrm{C}$, left panel). In contrast, incubation of HCT116/ Robo1 cells with hSlit2, but not PBS, $\mathrm{pH} 7.4$, increased Hakai binding to E-cad, which was neutralized by R5, but not by mIgG (Figure 4D, left panel). Cell lysates were also directly immunoblotted as sample loading controls (Figure 4A-4D, right panels). Notably, the identical results were obtained using another anti-Hakai $\mathrm{Ab}$ from Abcam (Supplementary information, Figure S3A-S3D).

Consistent with the recruitment of Hakai, HCT116/ Robo1/Slit2 cells manifested a significantly elevated level of E-cad ubiquitination as compared with HCT116/ $\mathrm{V}$ and HCT116/Robo1 cells (Figure 4E, upper panel). Conversely, SW620/shRobo1 cells displayed markedly diminished E-cad ubiquitination as compared with SW620/V cells (Figure 4F, upper panels). R5, but not mIgG, reduced E-cad ubiquitination in both HCT116/ 
A HCT116/Robo1/Slit2

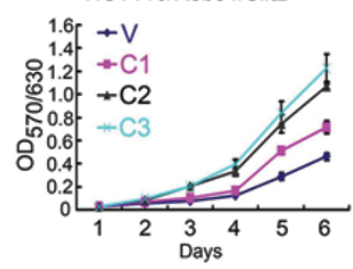

$\mathrm{E}$
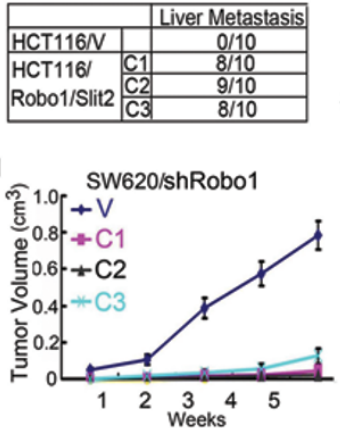

K HCT116/Robo1/Slit2

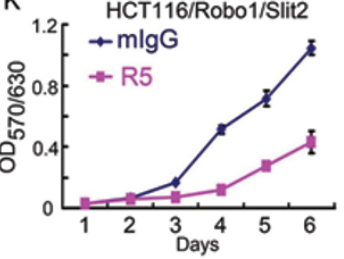

0

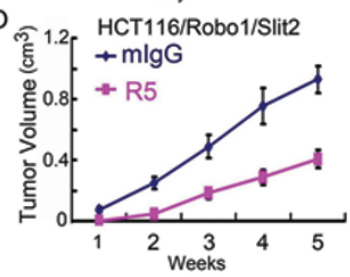

B HCT116/Robo1/Slit2
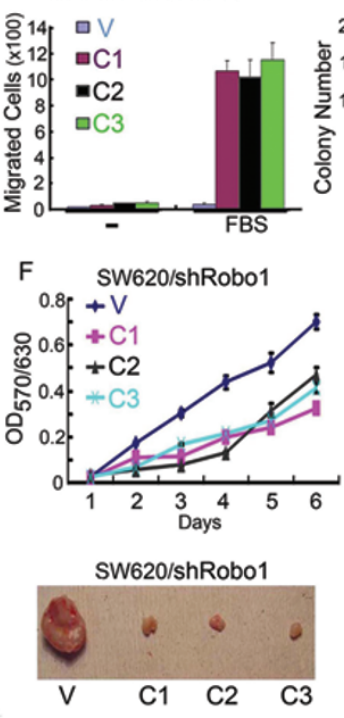

C HCT116/Robo1/Slit2 D

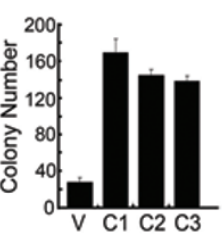

है 1.2 [ $+\mathrm{V}$

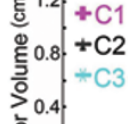

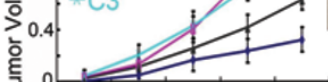

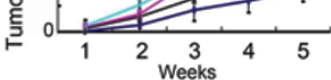
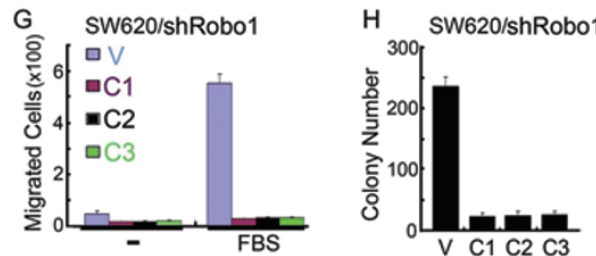

J
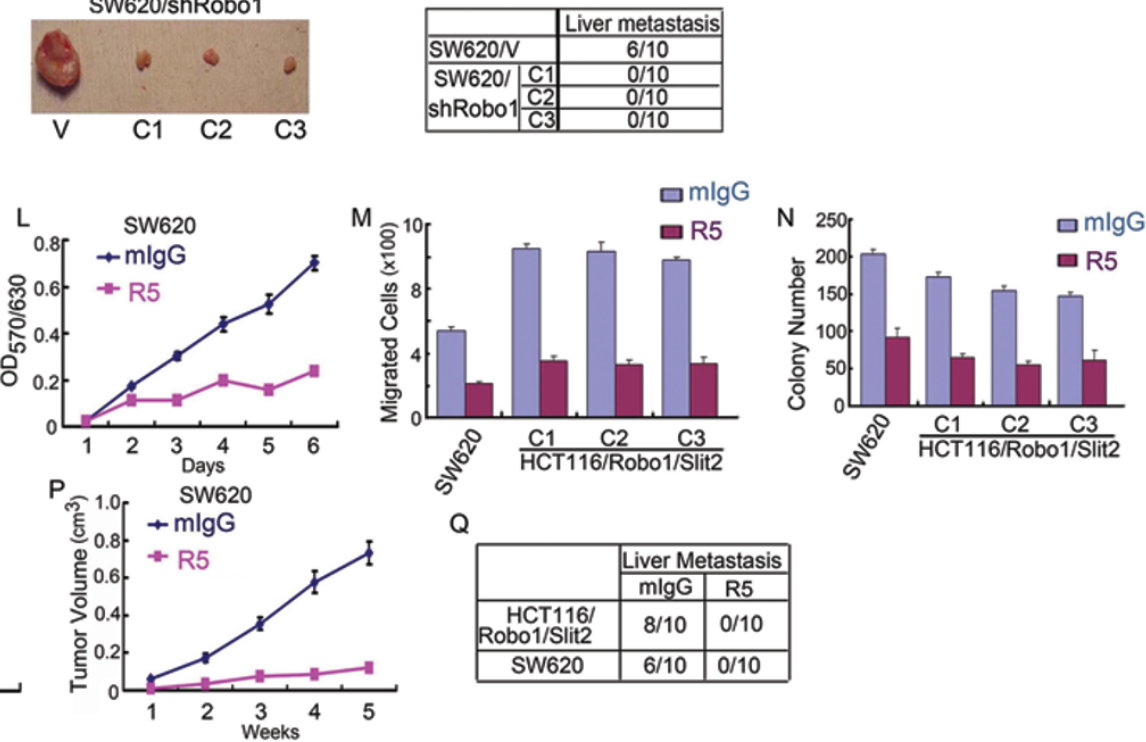

$\mathrm{Q}$

Figure 3 Slit-Robo signaling promotes intestinal epithelial cell carcinogenesis. (A, F, K and L) In vitro growth of HCT116/ $V$ and HCT116/Robo1/Slit2 cells (A), SW620/V and SW620/shRobo1 cells (F), HCT116/Robo1/Slit2 cells in the presence of mlgG or R5 (K) and SW620 cells in the presence of mlgG or R5 (L). Cell proliferation activities were measured as before [16]. (B, G and M) Migratory activity of HCT116/V and HCT116/Robo1/Slit2 cells (B), SW620/V and SW620/shRobo1 cells (G) or SW620 and HCT116/Robo1/Slit2 cells in the presence of mlgG or R5 (M). Cell migratory activities were determined using the Boyden chamber assay [16]. (C, H and N) Colony formation of HCT116/V and HCT116/Robo1/Slit2 cells (C), SW620/V and SW620/shRobo1 cells (H) or SW620 and HCT116/Robo1/Slit2 cells in the presence of mlgG or R5 (N). Cell transformation activities were measured by the colony formation assay. (D, I, $\mathbf{O}$ and $\mathbf{P}$ ) Average tumor volume in athymus nude mice subcutaneously inoculated with HCT116/V and HCT116/Robo1/Slit2 cells (D, left panel), SW620/V and SW620/shRobo1 cells (I, left panel) and HCT116/Robo1/Slit2 cells in the presence of mlgG or R5 (O) and SW620 cells in the presence of mlgG or R5 (P). Representative tumor mass of HCT116/V and HCT116/Robo1/Slit2 cells (D, right panel) and SW620/V and SW620/ shRobo1 cells (I, right panel). (E, $\mathbf{J}$ and $\mathbf{Q}$ ) Incidence of liver metastasis in athymus nude mice subcutaneously inoculated with HCT116/V and HCT116/Robo1/Slit2 cells (E), SW620/V and SW620/shRobo1 cells (J), and HCT116/Robo1/Slit2 and SW620 cells $(\mathbf{Q})$ in the presence of $\mathrm{mlgG}$ or R5. Results represent the mean \pm S.D. of three independent experiments (A and $\mathbf{C}$, $\mathbf{F}$ and $\mathbf{H}, \mathbf{K}$ and $\mathbf{N}$ ) or 10 animals for each group (D, I, $\mathbf{O}$ and $\mathbf{P}$ ).

Robo1/Slit2 and SW620 cells (Figure 4G, upper panels). In comparison, incubation of HCT116/Robo1 cells with hSlit2, but not with PBS, augmented E-cad ubiquitination, which was abolished by R5, but not by $\mathrm{mIgG}$ (Figure $4 \mathrm{H}$, upper panel). Immunoprecipitated E-cad was also immunoblotted for E-cad (Figure 4E-4H, lower panels). Our findings, thus, indicate that Slit-Robo signaling increases Hakai binding to E-cad, resulting in E-cad ubiquitination in colorectal epithelial cells. 
We next examined whether Slit2 binding to Robo1 enhanced E-cad localization in the lysosome. Using immunofluorescent staining of E-cad and LAMP-1, a lysosome marker, we found that E-cad was indeed localized exclusively on the cell-surface plasma membrane in HCT116/V cells (Figure 5A). In HCT116/Robo1/Slit2 cells, however, E-cad was re-distributed from the cellsurface plasma membrane to the cytoplasm, where it was partially co-localized with LAMP-1. Importantly, R5, but not $\mathrm{mIgG}$, restored the cell-surface localization of E-cad in HCT116/Robo1/Slit2 cells. In SW620 cells, Robo1 knockdown or R5 treatment resulted in the cell-surface localization of E-cad, in contrast to the cytoplasmic and lysosomal localization in untreated SW620/V cells (Figure 5B). Incubation of HCT116/Robo1 cells with hSlit2, but not PBS, also re-distributed E-cad from the cellsurface to the cytoplasmic and lysosomal compartments, which was prevented by R5, but not by $\mathrm{mIgG}$ (Figure 5C). Taken together, our results indicate that engagement of Robo1 by Slit2 targets E-cad to the lysosome through E-cad ubiquitination in colorectal epithelial cells.

\section{Slit-Robo signaling degrades E-cad through Hakai}

Given that Slit-Robo signaling increases E-cad ubiq- uitination and lysosomal localization, we next tested whether Slit-Robo signaling induces E-cad degradation by measuring the levels of E-cad following inhibition of protein synthesis. Cycloheximide (CHX), an inhibitor of protein synthesis, was incubated with cells for 0,6 and $12 \mathrm{~h}$. As suspected, E-cad degradation was observed in HCT116/Robo1/Slit2 cells, but not in HCT116/V or HCT116/Robo1 cells (Figure 6A). In contrast, E-cad was protected from degradation in SW620/shRobol cells as compared with SW620/V cells (Figure 6B). Incubation of HCT116/Robol cells with hSlit2, but not with PBS, also elicited E-cad degradation (Figure 6C). R5, but not $\mathrm{mIgG}$, prevented E-cad degradation in HCT116/Robo1/ Slit2 cells (Figure 6D), SW620 cells (Figure 6E) and HCT116/Robo1 cells in the presence of hSlit2 (Figure $6 \mathrm{~F})$. In contrast, E-cad degradation was prevented in cells with active Slit2-Robo1 signaling using chloroquin (an inhibitor for lysosomal degradation), MG132 (an inhibitor for proteasomal degradation) or chloroquin plus MG132 (Supplementary information, Figure S4A-S4C, upper panels). Notably, overexpression of Robol and Robo1 plus Slit2 in HCT116 cells, Robo1 knockdown in SW620 cells, or hSlit2 incubation of HCT116/Robo1, in the absence or presence of $\mathrm{mIgG}$ and $\mathrm{R} 5$, had no detect-
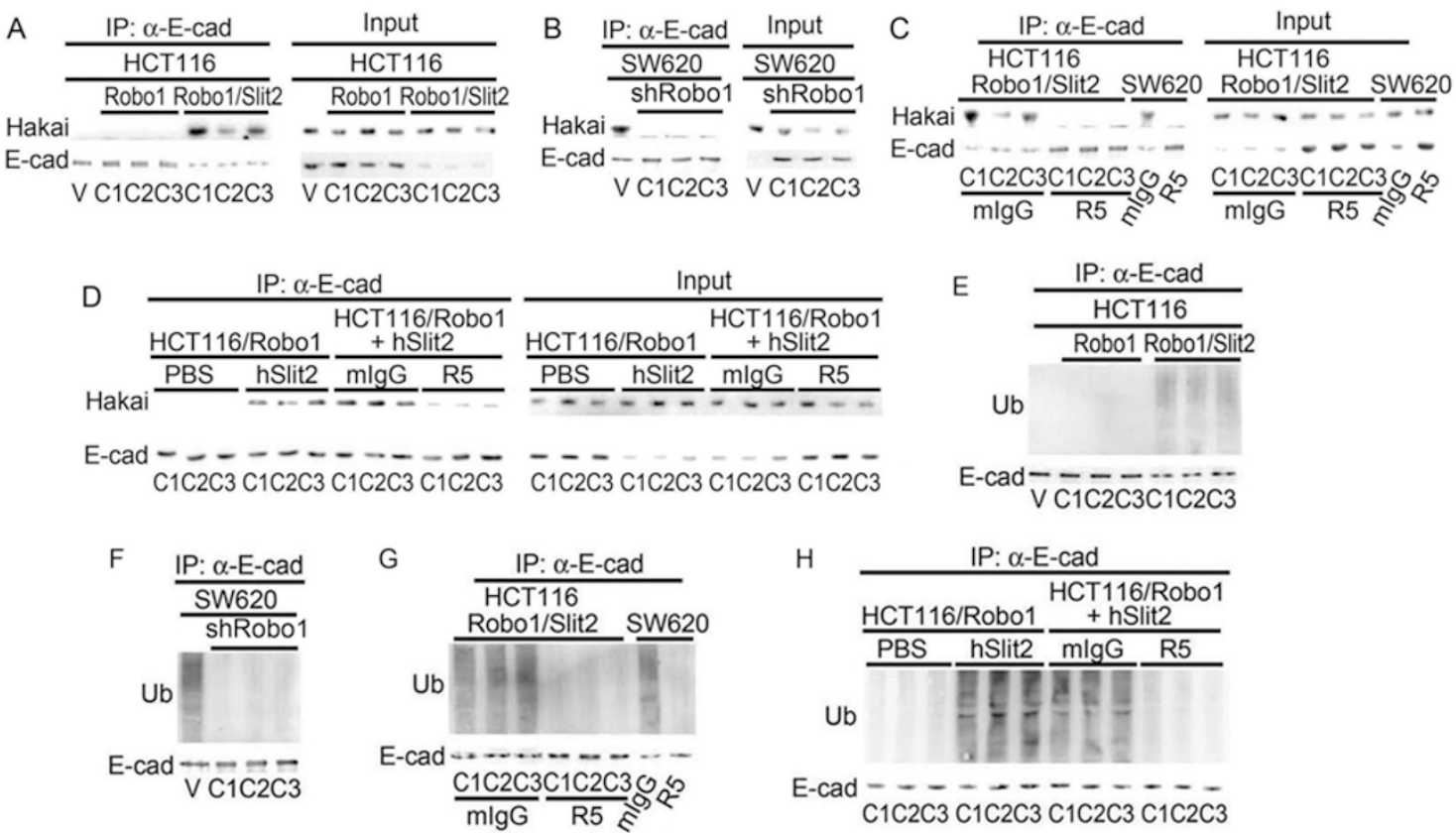

Figure 4 Slit-Robo signaling recruits Hakai to E-cad and induces E-cad ubiquitination. (A-D) Hakai binding to E-cad in HCT116/V, HCT116/Robo1, HCT116/Robo1/Slit2, SW620/V and SW620/shRobo1 cells in the absence or presence of mlgG, R5, PBS and hSlit2. E-cad was immunoprecipitated from the cell lysates followed by immunoblotting with the Abs to E-cad and Hakai. (E-H) E-cad ubiquitination in HCT116/V, HCT116/Robo1, HCT116/Robo1/Slit2, SW620/V and SW620/shRobo1 cells in the absence or presence of mlgG, R5, PBS and hSlit2. E-cad was immunoprecipitated from the cell lysates followed by immunoblotting with the ubiquitin and E-cad Abs. Ub, ubiquitin. Results are representative of at least three independent experiments. 
A

HCT116/

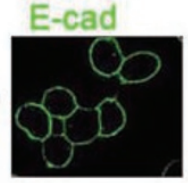

HCT116/

Robo1/Slit2

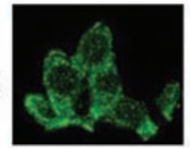
HCT116/
Robo1/Slit2
$\stackrel{+}{\mathrm{mlgG}}$

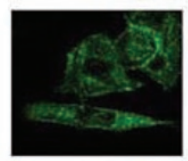
HCT116/
Robo1/Slit2 $\stackrel{+}{\mathrm{R} 5}$

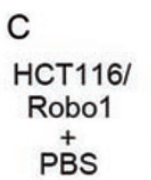
HCT116/
Robo1
$+$
hSlit2

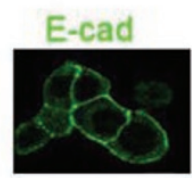

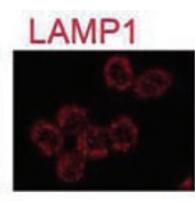
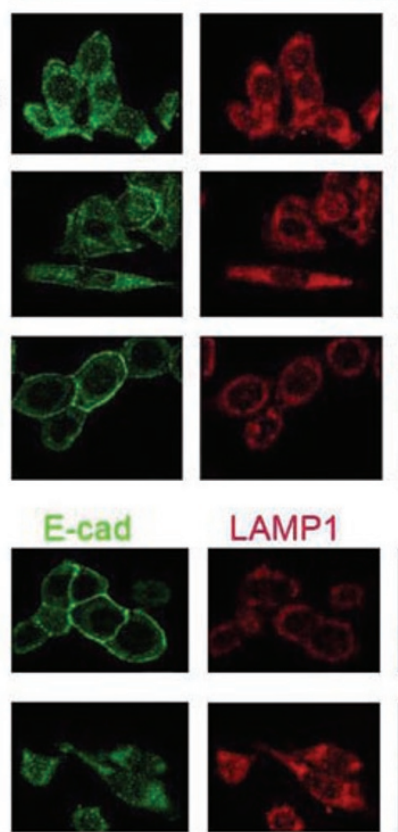
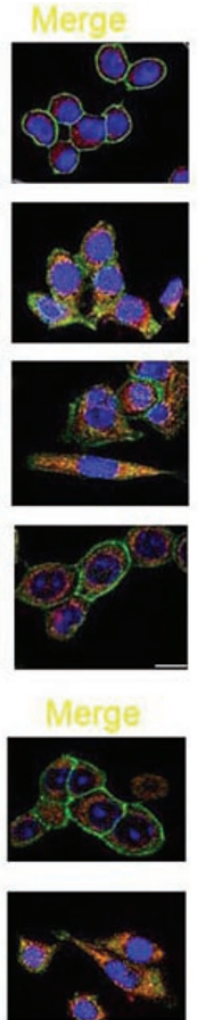

BW620/N
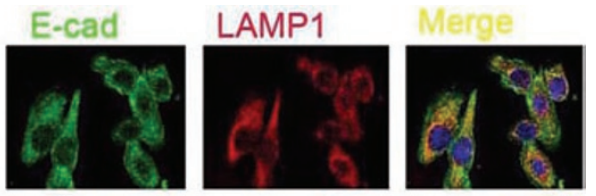

SW620/
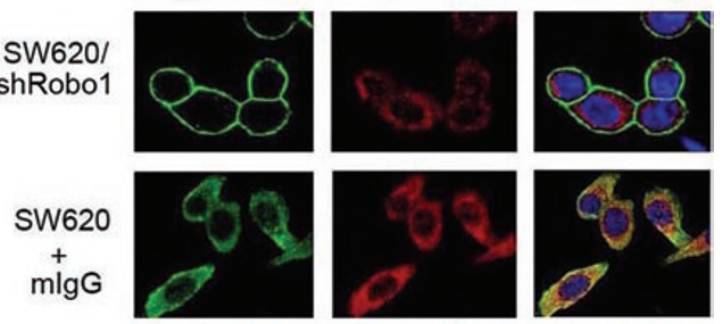

SW620

$\stackrel{+}{\mathrm{R} 5}$
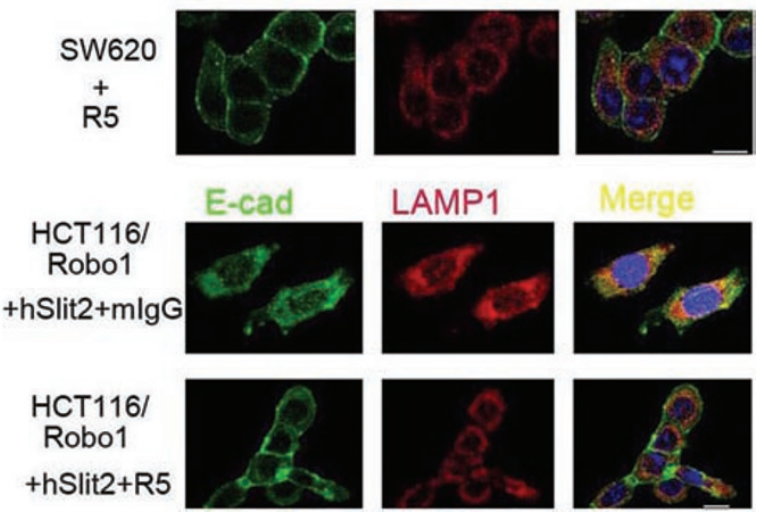

Figure 5 Slit-Robo signaling triggers E-cad lysosomal localization. (A) E-cad and LAMP-1 co-localization in HCT116/ $\mathrm{V}$ and HCT116/Robo1/Slit2 (C1) cells in the absence or presence of mlgG or R5. Endogenous E-cad and LAMP-1 were immunofluorescent stained with their respective Abs. (B) E-cad and LAMP-1 co-localization in SW620/V and SW620/ shRobo1 (C1) cells in the absence or presence of mlgG or R5. (C) E-cad and LAMP-1 co-localization in HCT116/Robo1 (C1) cells in the absence or presence of PBS, hSlit2, mlgG and R5. Bars, $10 \mu \mathrm{m}$. Results are representative of at least three independent experiments.

able effects on $\beta$-cat degradation (Supplementary information, Figure S4D, upper panels) and ubiquitination (Supplementary information, Figure S4E, upper panels). $\alpha$-Tubulin in the cell lysates (Supplementary information, Figure S4A-S4D, lower panels) and the immunoprecipitated $\beta$-cat (Supplementary information, Figure S4E, lower panels) were also immunoblotted for sample loading controls.

We next tested whether knockdown of endogenous Hakai could preserve E-cad from its ubiquitination and degradation induced by Slit-Robo signaling. Compared with the shRNA control, Hakai shRNA1 and 2 both potently inhibited Hakai expression (>95\%) in SW620 cells (Figure 7A, upper panel). As predicted, Hakai shRNA1 and 2, but not the shRNA control, drastically reduced E-cad ubiquitination immunoprecipitated from the lysates of HCT116/Robo1/Slit2 cells (Figure 7B, left upper panel), SW620 cells (Figure 7B, right upper panel) and hSlit2-treated HCT116/Robo1 cells (Figure 7C, upper panel). Compared with the shRNA control, Hakai
shRNA1 and 2 markedly reduced E-cad degradation in HCT116/Robo1/Slit2 cells (Figure 7D, upper left panel), SW620 cells (Figure 7D, upper right panel) and hSlit2incubated HCT116/Robo1 cells (Figure 7E, upper panel). In parallel, immunoprecipitated E-cad (Figure 7B and 7C, middle panels), endogenous Hakai (Figure 7B and 7C, lower panels and Figure 7D and 7E, middle panels) and $\alpha$-tubulin (Figure 7D and 7E, lower panels) were immunoblotted with their respective Abs. Consistently, Hakai shRNA1 and 2, but not the shRNA control, avidly switched HCT116/Robo1/Slit2 cells (Figure 7F), SW620 cells (Figure 7G) and hSlit2-incubated HCT116/Robo1 cells (Figure 7H) from the fibroblast-like morphology back to the epithelial-like morphology, with concomitant reduction of their cell migrations (Figure 7I). These results collectively demonstrate the significance of Hakai in mediating E-cad ubiquitination and degradation triggered by Slit-Robo signaling during colorectal epithelial cell carcinogenesis. 
Slit-Robo signaling fails to suppress E-cad transcription

We next explored whether Slit-Robo signaling might reduce E-cad protein levels through transcriptional repression. Using the HCT116 and SW620 cellular models, we examined whether Slit2 binding to Robo1 decreases the transcription of E-cad or increases its known transcriptional repressors. We co-transfected cells with an E-cad promoter luciferase reporter construct and a plasmid encoding a gene of interest or plain vector (V) (Supplementary information, Data S1). The snail plasmid was used as a positive control because it is known to transcriptionally downregulate E-cad [34]. HCT116/V, HCT116/Robo1 and HCT116/Robo1/Slit2 cells failed to appreciably suppress E-cad promoter activity compared with HCT116 cells transfected with the snail plasmid (Supplementary information, Figure S5A). As measured by qRT-PCR, HCT116/Robo1/Slit2 cells exhibited no significant decrease in the mRNA levels of E-cad or increase its transcriptional repressors Twist, Snail, Slug or Zeb2 compared with the mRNA levels in HCT116/ $\mathrm{V}$ cells (Supplementary information, Figure S5B). In addition, SW620/shRobo1 cells exhibited no obvious increase in mRNA levels of E-cad, Twist, Slug or Zeb2 compared with SW620/V cells (Supplementary informa- tion, Figure S5C). These data suggest that Slit-Robo signaling does not significantly reduce the levels of E-cad protein through transcriptional suppression of E-cad or its known transcriptional repressors.

\section{Slit-Robo signaling induces transformation of HEK293 cells}

To investigate whether Slit-Robo signaling can also induce transformation in epithelial cells other than colorectal epithelial cells, we established HEK293 cells stably overexpressing Robol or plain vector (293/Robo1 and 293/V). Notably, 293/Robo1 cells expressed a considerably high level of endogenous Slit2 (Figure 8A). Interestingly, 293/Robo1 cells manifested a remarkable fibroblast-like phenotype as shown by phase-contrast microscopy and immunofluorescent staining of $\alpha$-tubulin and $\beta$-actin (Figure 8B) with concomitant downregulation of E-cad, $\alpha$-cat and $\gamma$-cat in parallel to upregulation of N-cad and vimentin (Figure 8C). $\alpha$-Tubulin was immunoblotted in parallel as a sample loading control. Importantly, 293/Robo1 cells grew faster (Figure 8D) and migrated more rapidly (Figure $8 \mathrm{E}$ ) in vitro and formed larger tumors (Figure 8F) following subcutaneous inoculation in vivo. These results indicate that Slit-Robo sig-

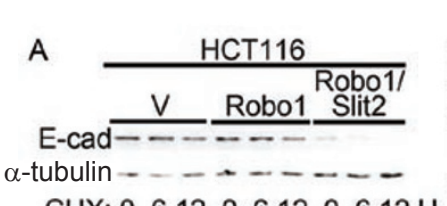

CHX: $061206120612 \mathrm{H}$
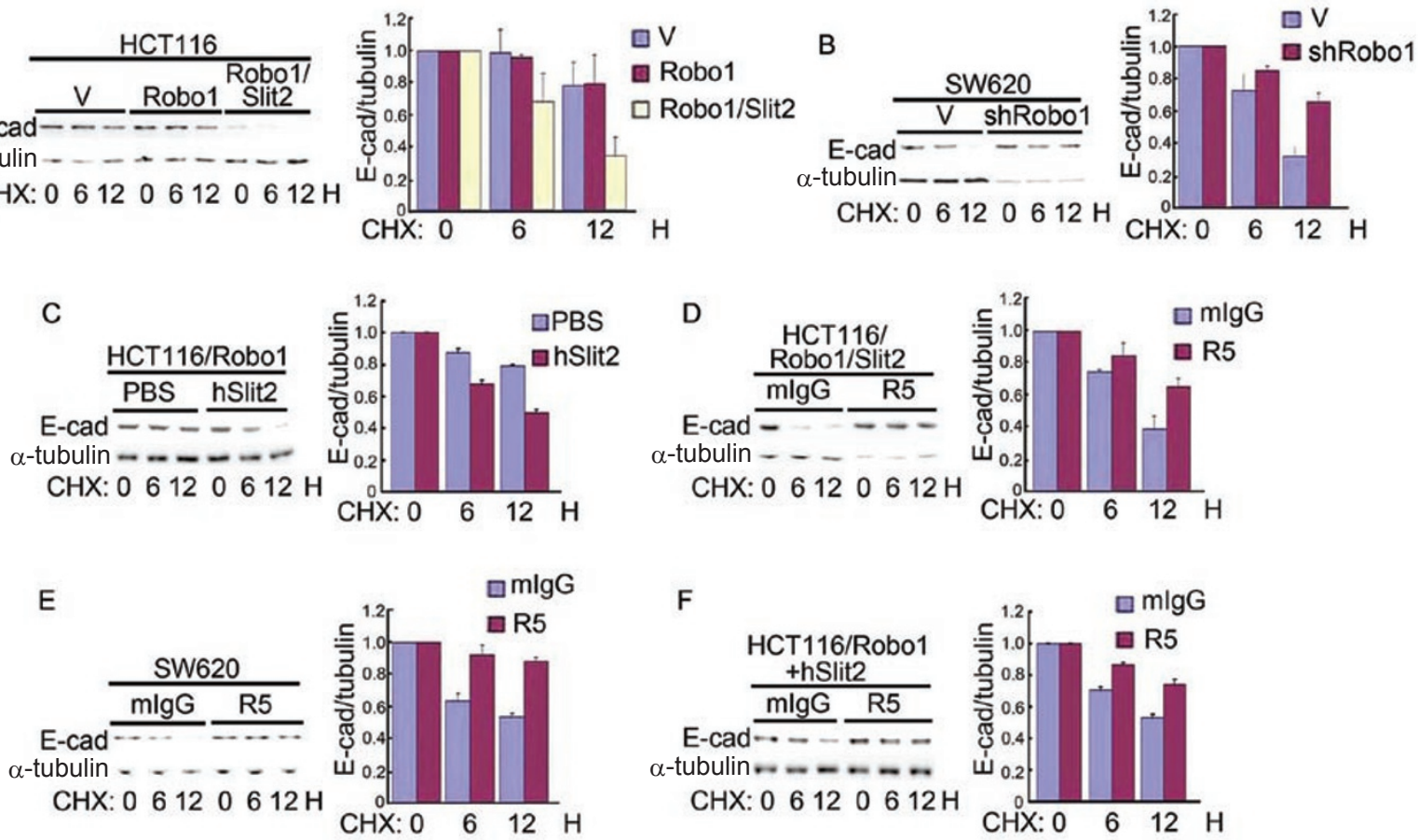

Figure 6 Slit-Robo signaling degrades E-cad. (A-F) HCT116/V, HCT116/Robo1, HCT116/Robo1/Slit2, SW620/V and SW620/shRobo1 cells were treated with CHX, in the absence or presence of mlgG, R5, PBS and hSlit2, for a period of time as indicated. Cell lysates were immunoblotted with the Abs to E-cad (left upper panels) and $\alpha$-tubulin (left lower panels) and a ratio of E-cad over $\alpha$-tubulin (right panels) was calculated. Results are representative of at least three independent experiments (left panels) or the mean \pm S.D. of three independent experiments (right panels).

www.cell-research.com | Cell Research 
B

A

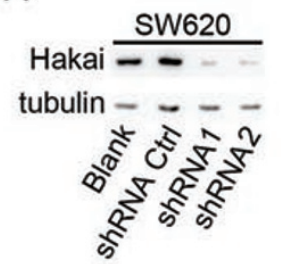

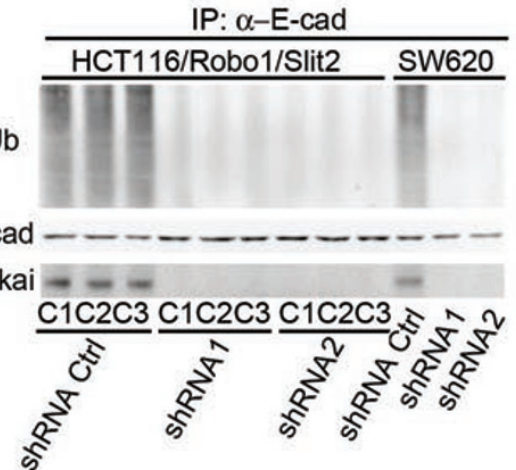

C

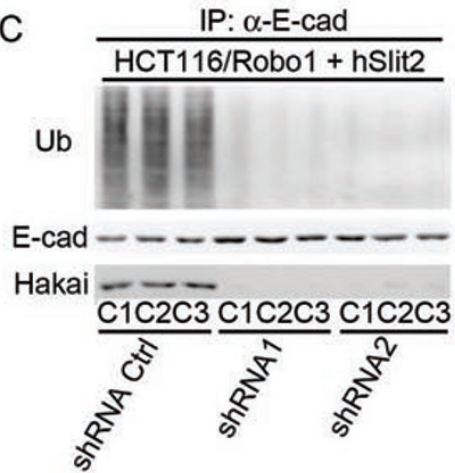

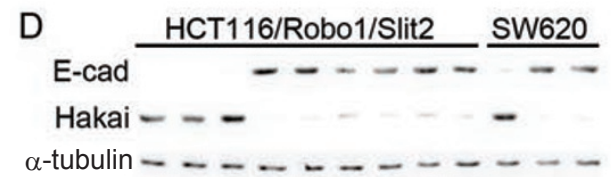

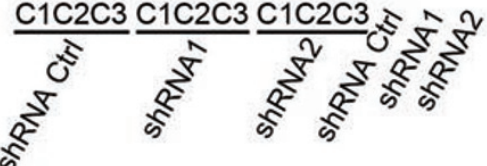

F

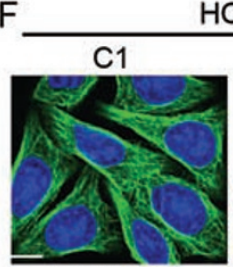

shRNA CtrI
HCT116/Robo1/Slit2

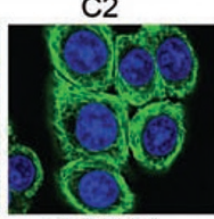

ShRNA1

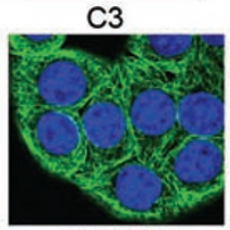

shRNA2
$\mathrm{H}$

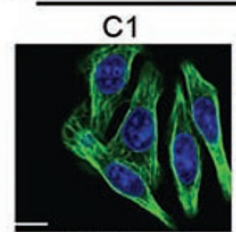

shRNA Ctrl
HCT116/Robo1 + hSlit2

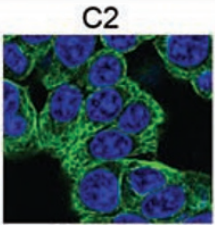

shRNA1

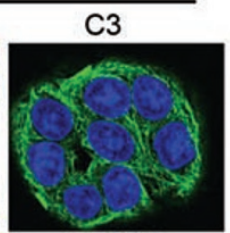

ShRNA2

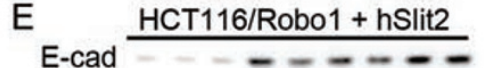

Hakai - - -
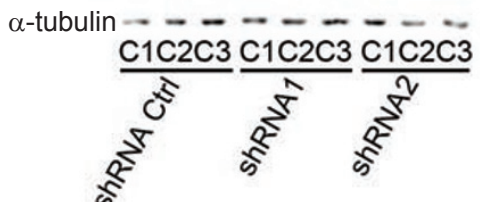

G

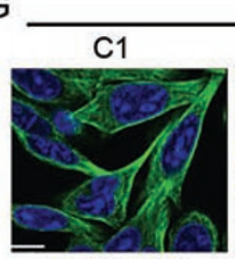

shRNA Ctrl
SW620

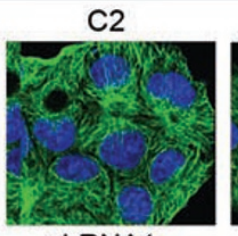

shRNA1

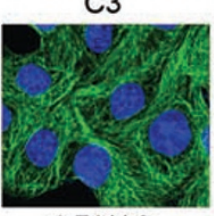

shRNA2

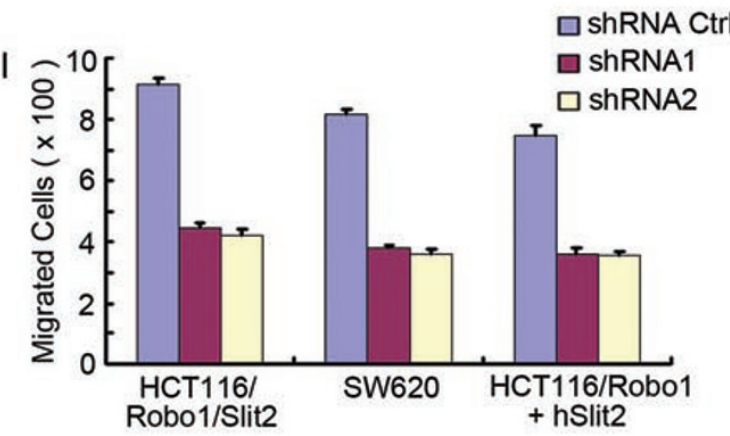

Figure 7 Roles of Hakai in E-cad ubiquitination/degradation and EMT. (A) Identification of Hakai shRNAs. SW620 cells were either not transfected (blank) or transfected with the plasmids of shRNA control and shRNA1 and 2 of Hakai. Transfectants were immunoblotted for Hakai and $\alpha$-tubulin. (B, C) Effects of Hakai knockdown on E-cad ubiquitination. HCT116/Robo1/ Slit2 and SW620 cells (B) or hSlit2-treated HCT116/Robo1 cells (C) were transfected with the plasmids of shRNA control and shRNA1 and 2 of Hakai followed by immunoblotting for ubiquilin (Ub, upper panels), E-cad (middle panels) and Hakai (lower panels). (D, E) Effects of Hakai knockdown on E-cad degradation. HCT116/Robo1/Slit2 and SW620 cells (D) or hSlit2-treated HCT116/Robo1 cells (E) were transfected with the plasmids of shRNA control and shRNA1 and 2 of Hakai followed by immunoblotting for E-cad (upper panels), Hakai (middle panels) and $\alpha$-tubulin (lower panels). (F-H) Effects of Hakai knockdown on cell morphology. HCT116/Robo1/Slit2 (F), SW620 cells (G) or hSlit2-treated HCT116/Robo1 cells (H) were transfected with the plasmids of shRNA control and shRNA1 and 2 of Hakai followed by immunofluorescent staining as above. (I) Effects of Hakai knockdown on cell migration. HCT116/Robo1/Slit2, SW620 cells or hSlit2-treated HCT116/Robo1 cells were transfected with the plasmids of shRNA control and shRNA1 and 2 of Hakai followed by the Boyden chamber assay for determination of cell migration. Bars, $10 \mu \mathrm{m}$. Results are representative of at least three independent experiments $(\mathbf{A}-\mathbf{H})$ or the mean \pm S.D. of three independent experiments (I). 
naling may act as a common mechanism for induction of transformation in normal epithelial cells in addition to its role in colorectal epithelial cells. Of course, this provocative hypothesis awaits further validation.

\section{Expression of Slit2 and Robol correlates with enhanced metastasis and shortened survival}

To examine whether Slit2 and Robo1 expression correlates with tumor growth and metastasis of colorectal epithelial cells, we analyzed the expression of these proteins in human colorectal carcinoma tissue samples. We generated an $\operatorname{IgG}_{2 \mathrm{a}} \mathrm{mAb}$ to the $\mathrm{N}$-terminal segment of human Slit2, S1, and an $\operatorname{IgG}_{1} \mathrm{mAb}$ to the C-terminal segment of rat Robo1, R4, as previously reported [16]. Here we examined the specificity of these mAbs. Lysates from HEK293 cells transfected with the plasmids of fulllength human Slit1 to 3 or plain vector was immunoblotted with S1. S1 bound to full-length Slit2 $(\sim 200 \mathrm{kDa})$ and the N-terminal segment of Slit2 $(\sim 150 \mathrm{kDa})$, as well as to human Slit1 and 3 (Supplementary information, Figure S2C). In addition, HEK293 cells were transiently transfected with the plasmids of human Robol and 2 fused with the MYC tag and human Robo 3 and 4 fused with the FLAG tag. The 9E10 binding to the MYC tag detected Robo 1 and 2 expression, whereas M2 binding to the FLAG tag detected Robo3 and 4 expression
(Supplementary information, Figure S2D). Interestingly, R4 bound to Robo1, but not to Robo2, 3 or 4. For negative controls, transfection of HEK293 cells with the plain vector resulted in no positive signals as measured by 9E10, M2 or R4. These results indicate that $\mathrm{S} 1$ is a panSlit $\mathrm{mAb}$, specifically recognizing all three Slit proteins, whereas R4 is specific for human Robol.

Using the S1 and R4 mAbs for pan-Slit and Robo1 immunofluorescent staining, we examined the tissue samples from 472 patients pathologically diagnosed with colorectal carcinoma (Figure 9A). Immunoreactivity for the pan-Slit antigens was seen in $17 \%$ more metastatic cancers than non-metastatic cancers while immunoreactivity for the Robol antigen was seen in $15 \%$ more metastatic cancers than non-metastatic cancers (Figure 9B). Interestingly, the frequency of both pan-Slit- and Robo1-positive staining in patients with metastatic cancer was $19 \%$ higher than that in non-metastatic cancer $(P<0.005)$. To verify these immunological findings and to clarify which Slit constitutes the pan-Slit antigen, we extracted RNAs from the pan-Slit- and Robo1-positive and -negative tumor samples ( $n=10$ for each group) and quantified Slit1-3 and Robo1-4 mRNAs by qRT-PCR. Compared with the pan-Slit and Robol-negative tumor group, Slit2 and Robo1 were significantly upregulated in the pan-Slit- and Robo1-positive tumor group (Figure

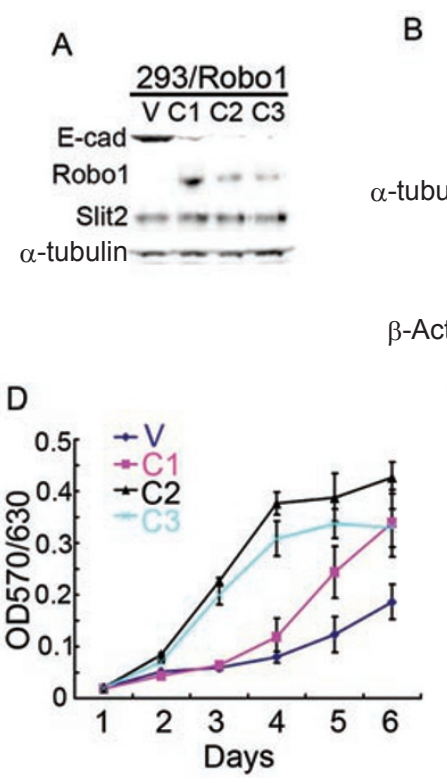

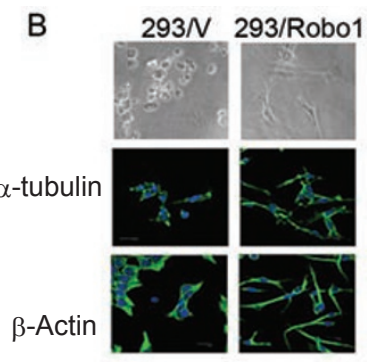

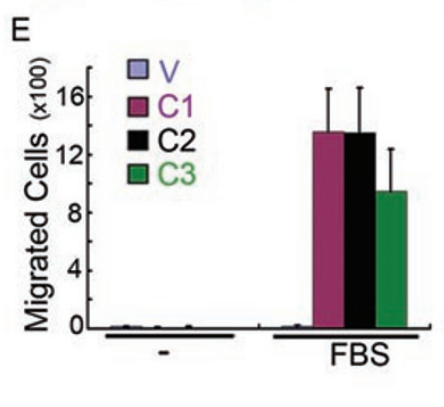

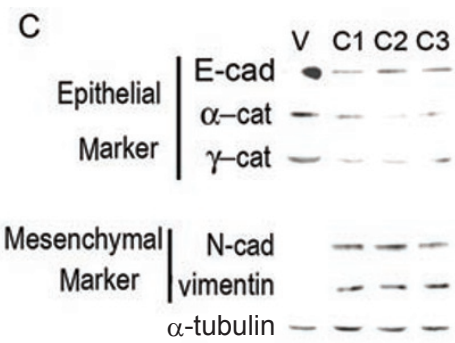

F

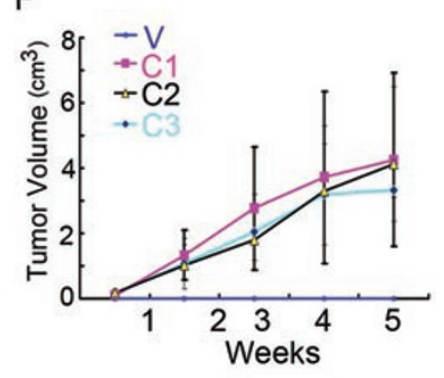

Figure 8 Expression of Robo1 induces transformation of HEK293 cells. (A) HEK293 cells stably transfected with the plain vector (293/V) or the plasmid encoding Robo1 (293/Robo1). C1-3, clone 1-3. (B) Cell morphology of 293/V and 293/Robo1 cells (C1) with or without immunofluorescent staining of $\alpha$-tubulin and $\beta$-actin. Cell nuclei were stained with DAPI. Bars, 10 $\mu \mathrm{m}$. (C) Expression of epithelial and mesenchymal markers in 293/N and 293/Robo1 cells. (D-F) In vitro proliferation (D) and migration (E) and in vivo tumor formation (F; $n=10$ for each group) of 293/V and 293/Robo1 cells. Results represent at least three independent experiments $(\mathbf{A}-\mathbf{C})$ or the mean \pm S.D. of three independent experiments (D-F). 
A

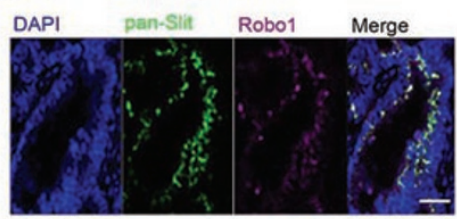

D

\begin{tabular}{|l|c|c|c|}
\hline $\begin{array}{l}\text { Colorectal } \\
\text { Cancer }(n=51)\end{array}$ & $\begin{array}{l}\text { pan-Slit } \\
\text { Robo1 }^{-} \text {( }(n=18)\end{array}$ & $\begin{array}{l}\text { pan-Slit } \\
\text { Robo1 }^{+} \text {( }(n=33)\end{array}$ & $p$ \\
\hline $\begin{array}{c}\text { Cell-surface } \\
\text { E-cad }\end{array}$ & $50 \%$ & $18.18 \%$ & $<0.025$ \\
\hline
\end{tabular}

F

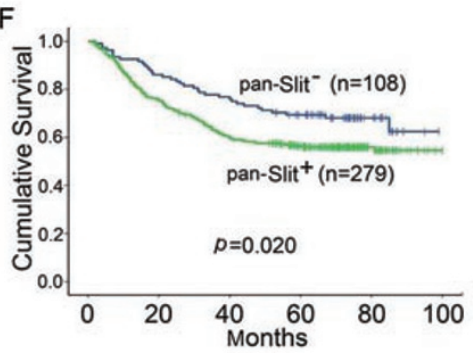

B

\begin{tabular}{|c|c|c|c|}
\hline $\begin{array}{c}\text { Colorectal } \\
\text { Cancer }(n=472)\end{array}$ & $\begin{array}{r}\text { Non-Metastasis } \\
(n=255)\end{array}$ & $\begin{array}{r}\text { Metastasis } \\
(n=217)\end{array}$ & $p$ \\
\hline pan-Slit $^{+}$ & $58.04 \%$ & $75.12 \%$ & $<0.005$ \\
\hline Robo1 $^{+}$ & $51.37 \%$ & $66.36 \%$ & $<0.005$ \\
\hline $\begin{array}{c}\text { pan-Slit } \\
\text { Robo1 }^{+}\end{array}$ & $41.96 \%$ & $60.37 \%$ & $<0.005$ \\
\hline
\end{tabular}

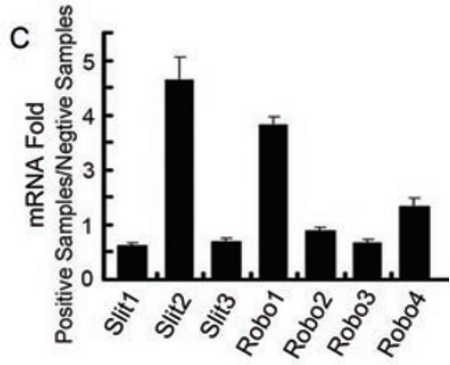

E
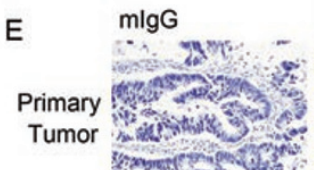

pan-Slit

Robo1
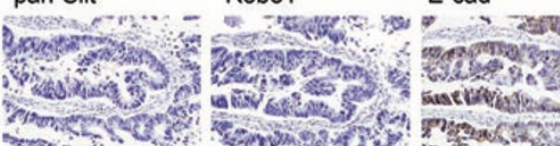

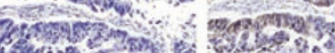

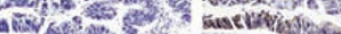

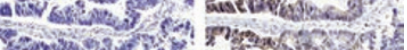

Metastatic

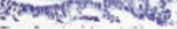

Tumor
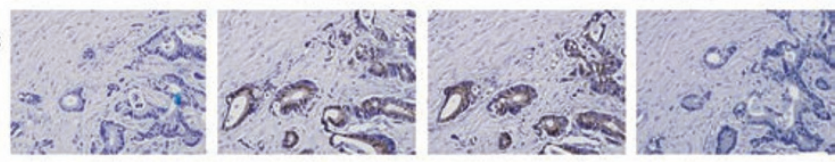

G

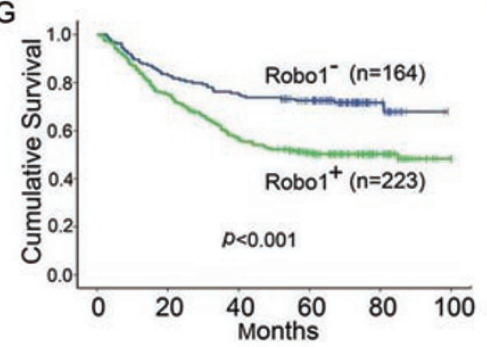

$\mathrm{H}$

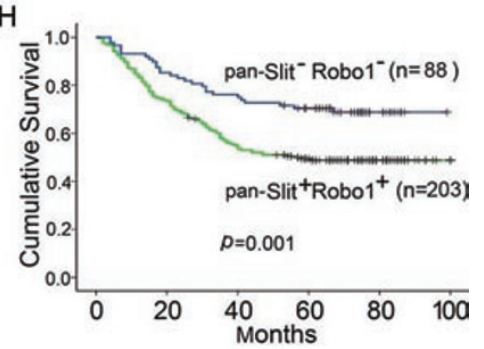

Figure 9 Clinical significance of Slit-Robo expression in colorectal carcinoma. (A) Immunofluorescent staining of panSlit, Robo1 and nucleus (DAPI) and their co-localization in the colorectal carcinoma tissue specimen. Bars, $0.1 \mathrm{mM}$. (B-D) Expression of pan-Slit and Robo1 antigens (B) and Slit1-3 and Robo1-4 mRNAs (C) and their association with cell-surface $\mathrm{E}$-cad (D) in the tissue specimen of non-metastatic and metastatic colorectal carcinoma. Pearson's $\chi^{2}$-test was used for statistical analysis (B, D). (E) Immunohistochemical staining of pan-Slit, Robo1 and E-cad in continuous sections of primary (upper panels) and metastatic (lower panels) colorectal carcinoma. Results are representative of at least 10 independent experiments. Bars, $0.1 \mathrm{mM}$. (F-H) Long-term survival curves of colorectal carcinoma patients based on the immunoreactivity of pan-Slit (F), Robo1 (G) and pan-Slit plus Robo1 (H).

9C). These mRNA expression data thus confirmed our immunohistochemical results using the $\mathrm{S} 1$ and $\mathrm{R} 4 \mathrm{mAbs}$. In addition, they demonstrated that Slit2, but not Slit1 or 3 , was likely the major antigen detected by the $\mathrm{S} 1 \mathrm{mAb}$. Notably, Robo4 mRNA was also slightly upregulated. Taken together, these findings indicate that the increased frequency of pan-Slit (mainly Slit2) and/or Robo1 expression may predict the metastasis or prognosis of colorectal carcinoma.

We next investigated whether pan-Slit and Robo1 expression was associated with the expression of E-cad in 51 cases of colorectal carcinoma (Figure 9D). As expected, the pan-Slit and Robo1 antigens negatively correlated with cell-surface E-cad. Using continuous tissue sectioning, we found that the epithelial layer of human colorectal primary tumors expressed cell-surface E-cad when lacking pan-Slit antigen and Robol (Figure 9E, upper panel). In contrast, the tissue of colorectal metastatic tumors expressed pan-Slit antigen and Robol when losing E-cad expression (Figure 9E, lower panel). These findings in human samples suggest that Slit-Robo signaling may reversibly correlate to E-cad expression during the pathogenesis of tumor growth and metastasis.

We further evaluated whether the pan-Slit and Robo1 immunoreactivities correlated with overall survival in 387 patients with colorectal carcinoma. As suspected, we found that the pan-Slit antigen $(P=0.020$; Figure 9F), the Robo1 antigen $(P<0.001$; Figure $9 \mathrm{G})$, and the panSlit plus Robol antigens $(P=0.001$; Figure $9 \mathrm{H})$ all inversely correlated with overall survival, highlighting the clinical importance of Slit-Robo signaling in determining the prognosis of colorectal carcinoma. 


\section{Discussion}

This study demonstrates that engagement of Robol by Slit2 recruits Hakai to E-cad, culminating in E-cad ubiquitination and lysosomal degradation (Figure 10). Experimentally, ectopic expression of Slit2 and Robo1 and treatment of Robo1-positive cells with recombinant Slit2 induced an EMT-like phenotype, while knockdown of Robo1 or mAb blockade of Slit2 binding to Robo1 triggered a MET-like phenotype. Notably, our finding that expression of Robol triggers a malignant transformation in Slit2-positive HEK293 cells is fully consistent with the essential roles of Slit-Robo signaling during cell morphogenesis and organ development. Our findings, thus, have implications for the fundamental significance of Slit-Robo signaling in the malignant transformation of epithelial cells derived from different organs in addition to the colorectal intestine. Importantly, these experimental results are consistent with our clinical findings in 472 cases, in which metastatic human colorectal carcinoma tissue samples showed an increase in pan-Slit (mainly Slit2) and Robo1 expression compared to non-metastatic tissue samples. Remarkably, the pan-Slit and Robo1 immunoreactivities inversely correlated with overall survival in patients diagnosed with colorectal carcinoma, further underscoring the clinical significance of Slit-Robo signaling in the pathogenesis and prognosis of colorectal carcinoma.

During assembly of the Drosophila heart tube, two types of progenitor cells, cardioblasts and pericardial cells, are generated within bilateral fields in the lateral mesoderm. These cells align into rows and coordinately migrate, in an intricate manner, to the dorsal midline of the embryo, where they merge and assemble into a beating linear heart with a central lumen. Notably, cardioblasts are specifically adhered at dorsal and ventral attachment points in an E-cad-dependent manner, where
Slit and Robo transcripts are absent. In contrast, the apical surface of cardioblasts facing toward the lumen expresses Slit and Robo, where E-cad is absent. Overexpression of Slit and Robo thus results in ectopic lumen formation. However, Robo mutant embryo fails to form a cardiac tube between adherent contralateral cardioblasts due to aberrant expression of E-cad. It is thus apparent that Slit-Robo signaling inhibits E-cad-mediated cell adhesion, which is obligatory for lumen formation between apical cardioblasts [40-44]. In this context, our finding that the autocrine Slit-Robo interaction downregulates E-cad may plausibly explain the underlying molecular mechanism for the action of Slit-Robo signaling in cardiac cell polarity and tube lumen formation during development of Drosophila heart.

By promoter region $\mathrm{CpG}$ island hypermethylation and allele loss, SLIT2 is reportedly inactivated in various cancer cell lines and primary lung, breast, intestinal, cervical and hepatocellular carcinomas, gliomas and lymphocytic leukemia, suggesting that SLIT2 may act as a candidate tumor suppressor gene [45-51]. Notably, Slit2 is also found to enhance E-cad-mediated cell-cell adhesion of breast cancer MCF-7 cells [52] and to increase E-cad expression through downregulation of its transcriptional repressor, Snail, in lung cancer [53].

However, SLIT2 promoter hypermethylation does not significantly associated with the clinical and pathological features of neuroblastoma, Wilms' tumor and renal cell carcinoma [54]. Hypermethylation of several CpG islands is frequently found in normal lung and breast tissues of cancer patients $[55,56]$. Interestingly, the inhibitors of DNA methylation and histone deacetylases fail to reactivate SLIT1-3, ROBO1 and $\mathrm{ROBO} 3$ expression in cervical cancer, implicating a complex mechanism involved for the observed inactivation of these gene products [57]. Notably, in sharp contrast to the previous report [52], breast cancer MCF-7 cells are typically

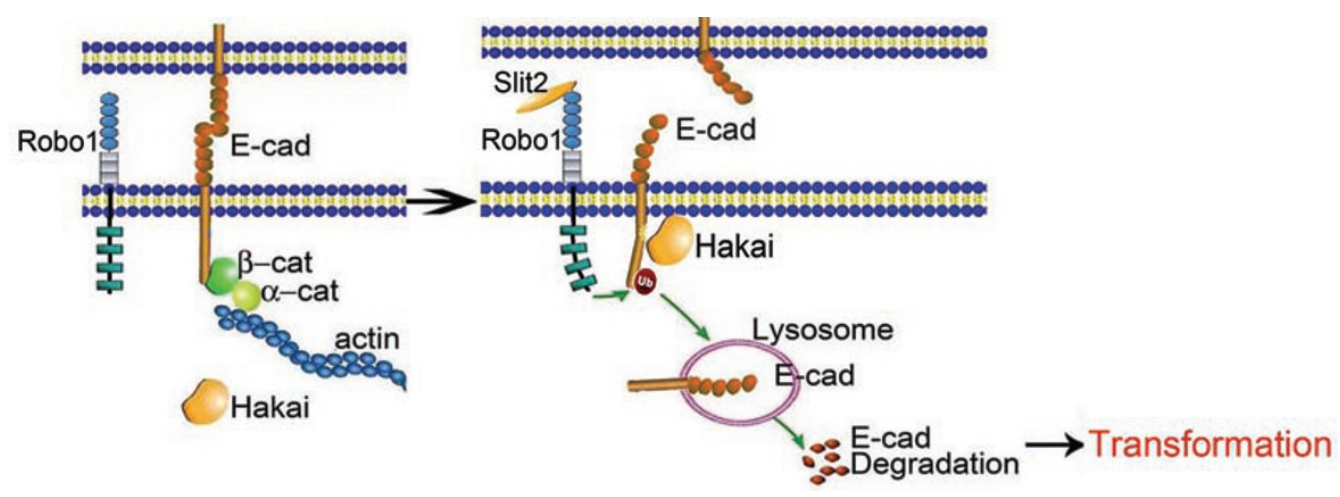

Figure 10 Schematic illustration of our proposed model. 
epithelial-like in morphology and express a high level of E-cad that is concentrated at the cell-cell contact [33, 58-61]. Furthermore, the engagement of Robo1 by Slit2 degrades N-cad and enhances motility of neuronal cells $[62,63]$. The experimental findings of this study also provide compelling evidence for induction of EMT-like phenotype through downregulation of E-cad in colorectal epithelial cells by autocrine Slit-Robo signaling.

These seemly paradoxical findings for the biological roles of Slit-Robo signaling in carcinogenesis are apparently reminiscent to the fascinating actions of these guidance cues in regulating both repulsive and attractive migration of such mammalian cells as neuronal, leukocytic, endothelial and cancerous cells [6, 64, 65], attesting to the context dependent and multifaceted roles of Slit-Robo signaling in cell migration and perhaps also in carcinogenesis. We speculate that in response to Slit2, it could be a combinatory array of intracellular signaling mechanisms downstream of cell-surface Robo1 that may not only regulate repulsive versus attractive cell migration, but also determine EMT-like versus MET-like phenotype of epithelial cells, governed by the temporal and spatial instruction and priming from neighboring cells at the given microenvironments. In this regard, Slit-Robo signaling may resemble TGF- $\beta$ [66], bone morphogenetic proteins [67], hepatic growth factor [68], Wnt/Frizzled $[69,70]$, estrogen [59] and microRNAs (miR-200 family and miR-205) [71] for reversible epithelial to mesenchymal-like transition and cell migration. Importantly, phenotypic plasticity of epithelial cells is obligatory for tube formation during development and for metastasis during carcinogenesis [23-26].

Intracellular molecules downstream of Robo signaling have emerged as key regulators for its biological functional activities on the targeting cells. Robo utilizes a variety of different signaling components in different cell types, such as Mena/Ab1 [5, 72], Slit-Robo GTPase-activating protein 1 [73] and phosphoinositol-3 kinase [16]. In this context, it should be mentioned that no tyrosine phosphorylation of immunoprecipitated Robol was detected in the lysates of HCT116/Robo1/Slit2 or SW620 cells (data not shown) and no c-Abl binding to Robo1 [62, 63] was found in SW620 cells (data not shown). These results apparently suggest that the downstream components of Slit-Robo signaling may be overlapping but distinct between colorectal epithelial cells and neuronal/ retinal cells.

Previous studies by us and others have shown that Slit-Robo signaling critically participates in vasculogenesis, angiogenesis and lymphangiogenesis [16-22, 74-78]. For instance, a variety of cancer cells secrete Slit2, which binds to Robo1 expressed on vascular endothelial cells and mediates tumor angiogenesis via a paracrine mechanism [16-19, 21]. In addition, we have recently demonstrated that Slit2 binds to Robo1 expressed on lymphatic endothelial cells, which induces lymphangiogenesis and lymphatic metastasis [22]. This study demonstrates the existence of an autocrine mechanism for mediating tumor cell-tumor cell signaling during growth and metastasis of colorectal carcinoma. On the basis of these observations, we propose that Slit-Robo signaling may act through both paracrine and autocrine mechanisms to synergistically promote the malignant transformation of colorectal epithelial cells. In this context, the profoundly potent effects of R5, which blocks the Slit2-Robo1 interaction, on in vivo tumor growth and liver metastasis of HCT116/ Robo1/Slit2 cells and SW620 cells can be partially explained by its inhibition of paracrine tumor angiogenesis mediated by Slit-Robo signaling. Although R5 markedly inhibited in vitro growth of HCT116/Robo1/Slit2 cells and SW620 cells in the present study, it did not inhibit human malignant melanoma A375 cells in a previous study (see Figure 3I of reference [16]). Overexpression of Slit2 did not promote A375 cell growth in culture (see Supplementary information, Figure S1B of reference [16]). These seemingly contradictory results are because of the lack of detectable Robo1 expression in A375 cells and the absence of the autocrine Slit-Robo interaction (see Figure 1H of reference [16]). Therefore, it is reasonable to conclude that blockade of Slit-Robo signaling may potently inhibit tumor angiogenesis and effectively prevent malignant transformation for suppression of tumor growth and metastasis in colorectal carcinoma. As a result, we believe that inhibitors of Slit-Robo signaling warrant clinical testing for treating colorectal carcinoma.

\section{Material and Methods}

\section{Plasmids, antibodies and chemicals}

Monoclonal Abs specific for human Robo1, Slit2, pan-Slit as well as R5 and its isotype-matched mouse $\operatorname{IgG}_{2 b}$ control were prepared and characterized as previously described [16]. The plasmid of snail (16218) was purchased from Addgene; human Slit1 (KIAA0813) and Slit3 (KIAA0814) were obtained from KAZUSA DNA Research Institute; human Robo2-4 were purchased from OriGene. The control shRNA (RHS4430-98524293) and shRNA1 and 2 (RHS4430-98714486 and RHS4430-98841956) of Hakai were purchased from Open Biosystems. Primary mAbs to E-cad, $\alpha$-cat, $\beta$-cat, $\gamma$-cat and $\mathrm{N}$-cad were obtained from BD Biosciences. Monoclonal Abs to vimentin, $\alpha$-tubulin, $\beta$-actin, $\mathrm{CHX}$, chloroquin and MG132 were prochased from Sigma. Hakai, ubiquitin and LAMP-1 Abs were purchased from Santa Cruz Biotechnology. The second anti-Hakai Ab (ab91185) was purchased from Abcam.

Cell cultures, stable cell lines and treatment

Human colorectal carcinoma cell lines SW620 (CCL-227), 
SW480 (CCL-228), SW48 (CCL-231), HCT116 (CCL-247), HT29 (HTB-38), LS180 (CL-187), LS174T (CL-188), DLD1 (CCL-221), Lovo (CCL-229) and Caco (HTB-37) as well as HEK293 cells (CRL-1573) were purchased from American Type Culture Collection (ATCC). They were cultured according to the recommendations from ATCC.

For establishment of stable cell lines, HCT116 and HT29 cells were transfected with the plasmids of full-length, wild-type Robol (HCT116/Robo1 or HT29/Robo1 cells), Slit2 (HCT116/Slit2 or HT29/Slit2 cells) or the plain vector (HCT116/V o HT29/V cells), using Lipofectamine 2000 (Invitrogen). They were selected by 0.8 mg/ml G418 (GIBCO BRL). HCT116/Robo1 and HT29/Robo1 cells were further transfected with the plasmid of Slit2 (HCT116/ Robo1/Slit2 or HT29/Robo1/Slit2 cells) and selected by $0.5 \mathrm{mg} /$ $\mathrm{ml}$ hygromycin B (Sigma). SW620 cells were transfected with the plasmid of Robo1 shRNA (SW620/shRobo1) or the plain vector and selected by $1 \mathrm{mg} / \mathrm{ml} \mathrm{G} 418$. Stable single-cell clones were collected for HCT116/Robo1 or HT29/Robo1 cells (C1, C2 and C3), HCT116/Slit2 or HT29/Slit2 cells (C1, C2 and C3), HCT116/Robo1/Slit2 or HT29/Robo1/Slit2 cells (C1, C2 and C3), HCT116/V or HT29/V cells, SW620/shRobo1 cells (C1, C2 and C3) and SW620/V cells. Human Robo1 shRNA was inserted into the pSuper-GFP vector (OligoEngine) with the following targeting sequences: 5'-GGCAUAUUUGGAAGUUACAtt-3' (sense) and 5'-UGUAACUUCCAAAUAUG CCtt-3' (antisense).

For inhibition of endogenous Slit2 binding to Robo1, HCT116/ Robo1/Slit2, SW620 and HT29/Robo1/Slit2 was incubated with $2 \mu \mathrm{g} / \mathrm{ml} \mathrm{mIgG}$ or R5 at $37{ }^{\circ} \mathrm{C}$ for $24 \mathrm{~h}$. Alternatively, HCT116/ Robol cells were incubated with PBS or $0.5 \mu \mathrm{g} / \mathrm{ml} \mathrm{hSlit} 2$ in the absence or presence of $2 \mu \mathrm{g} / \mathrm{ml} \mathrm{mIgG}$ or R5 at $37{ }^{\circ} \mathrm{C}$ for $24 \mathrm{~h}$.

\section{Immunoblotting, immunoprecipitation and GST pull-down}

Immunoblotting, immunoprecipitation and GST pull-down were carried out as described previously [77]. For subcellular fractionation, cell-surface, cytoplasmic and nuclear extracts were prepared using Qproteome Cell Compartment Kit (Qiagen). For inhibition of E-cad binding to Hakai, $3 \mu \mathrm{g}$ GST-E-cad were incubated with the SW620 cell lysates in the absence or presence of $3 \mu \mathrm{g}$ His-CC3 at $4{ }^{\circ} \mathrm{C}$ for $6 \mathrm{~h}$. Quantitative analyses of immunoblotting signals were obtained via densitometry analysis using LAS4000 Image Software (Fuji Film).

\section{Immunofluorescent staining}

Cells were fixed with $4 \%$ of paraformaldehyde and permeabilized with $0.05 \%$ of Triton X-100 in PBS at room temperature for $20 \mathrm{~min}$. Samples were blocked with $1 \%$ of bovine serum albumin (Sigma) and incubated with appropriate primary antibody at $37^{\circ} \mathrm{C}$ for $1 \mathrm{~h}$. After washing extensively, they were incubated with Alexa Fluor-488 goat anti-mouse IgG (Invitrogen) or Fluor-Cy3 goat anti-rabbit IgG (Jackson Immunoresearch) at $37^{\circ} \mathrm{C}$ for $1 \mathrm{~h}$. Cells were then washed and mounted for observation under a scanning confocal microscope (TCS SP2 Leica).

\section{$R T-P C R$}

Total RNA was extracted using the Absolutely RNA Miniprep Kit (Stratagene). For isolation of RNAs from paraffin-embedded tissues, each sample contained microdissected 30 pieces of $6 \mu \mathrm{m}$ thick paraffin-embedded colorectal continuous slices. Following treatment with xylene at $55^{\circ} \mathrm{C}$, it was washed in absolute ethanol, dried and resuspended in the lysis buffer (Stratagene). After ho- mogenizing thoroughly, it was digested with Protein K $(0.4 \mathrm{mg})$ $\mathrm{ml})$ at $45{ }^{\circ} \mathrm{C}$ overnight, and total RNA was extracted as above. They were reverse transcribed and the resulting cDNA was used for PCR using the SYBR-Green Master PCR Mix (Applied Biosystem) in triplicates. All $\mathrm{RT}^{2}$ qPCR Primer pairs were purchased from SABiosciences. PCR and data collection were performed on the Mx3000 qPCR System (Stratagene). All quantitations were normalized to an endogenous $\beta$-actin control. The relative quantitation value for each target gene compared to the calibrator for that target is expressed as $2-(\mathrm{Ct}-\mathrm{Cc})(\mathrm{Ct}$ and $\mathrm{Cc}$ are the mean threshold cycle differences after normalizing to $\beta$-actin). The relative expression levels of samples are presented using a semi-log plot.

\section{Construction and expression of recombinant hSlit 2 and hRo- bol-Fc}

For construction of hSlit2, the forward primer 5'-ACCTTCTAGAATGCGCGGCGTTGGCTGGC-3' and the reverse primer 5'-GCAGCGGCCGCTCAGTGATGATGATGATGATGATCTGCCATTTCTCCAGGACC-3' were used to amplify the insert (1-2 670 bp) from human Slit2 cDNA. After restriction enzyme digestion with $X b a \mathrm{l} / B g I I I$, it was ligated into the pVL1393 vector (BD Pharmingen), encoding the 1-890 amino acid residues of human Slit2, including the first to fourth leucine-rich repeats and first to sixth epidermal growth factor-like domains. For construction of hRobo1-Fc, the first to fifth immunoglobulin-like segment (1-1 760 bp) was amplified from human Robol cDNA (Origene) using the forward primer 5'-GGCCAAGCTTATGAAATGGAAACATGTTCC- ${ }^{\prime}$ ' and the reverse primer 5'-TCCACGGAATTCAAATTTGGTTGCC-3'. The PCR product was digested with HindIII and EcoRI, which was subcloned into the pEGFP-N1 vector (BD Pharmingen) to generate pEGFP-N1-hRobo1. The human immunoglobulin heavy chain (714 bp) was amplified from pAck-CH3 (US Biologicals) using the forward primer 5'-AACCGTGAATTCCGTGGACAAGAGAGTTGAGCC-3' and the reverse primer 5'-TACGGGTCGACTCATTTACCCGGAGACAGGG-3'. The PCR product was digested with EcoRI and SalI and subcloned into pEGFP-N1-hRobo1 to generate pEGFP-N1-hRobo1-Fc, which was used as the template to PCR the hRobol-Fc segment using the forward primer 5'-AGGCGGCCTCTAGAATGAAATGGAAACATGTTCC-3' and the reverse primer 5'-TACGGGCGGCCGCTCATTTACCCGGAGACAG-3'. The PCR product was digested by $X b a \mathrm{I}$ and NotI, and ligated to the pVL1393 vector (BD Pharmaingen) for pVL1393-hRobo-Fc. The authenticity of these constructs was confirmed by DNA sequencing. Recombinant hSlit2 and hRobo1-Fc were expressed in Sf9 cells and purified as previously described [79].

\section{Determination of promoter activities}

For measurements of E-cad promoter activity, the plasmid encoding the E-cad promoter luciferase reporter was co-transfected with the $\beta$-galactosidase plasmid into HCT116 cells using Lipofectamine 2000 (Invitrogen) [80].

\section{Cell proliferation assay}

Cells including all transfectants were grown to exponential phase and their proliferation activities were determined [16, 80].

\section{Boyden chamber assay}

A cell migration assay using a 48 -well micro-chemotaxis chamber (Neuro Probe) was performed [16]. 


\section{Colony formation assay}

For determination of cell transformation, a bottom layer of $0.6 \%$ agar noble in DMEM without serum was first placed onto a sixwell plate. Cells were seeded in $0.3 \%$ top agar noble in DMEM with $10 \%$ FBS. Fresh top agar was added after 10 days. Colonies that had formed were counted after 3 weeks.

\section{Xenograft model of tumor growth and metastasis}

Cells were resuspended at $1 \times 10^{7}$ cells $/ \mathrm{ml}$ and an aliquot of $0.1 \mathrm{ml}$ cell suspension was injected subcutaneously into athymic nude mice [16]. For antibody inhibition experiments, mice bearing HCT116/Robo1/Slit2 or SW620 cell tumors were treated with intraperitoneal injections of $\mathrm{R} 5$ or $\mathrm{mIgG}_{2 \mathrm{~b}}$ control twice per week (1 $\mathrm{mg}$ per injection). For metastasis measurements, mouse livers and lungs were collected after subcutaneous inoculation of cells for 5 weeks followed by counting of tumor nodules (routinely more than five nodules per liver or lung).

\section{Immunohistochemical staining}

Patient tissue samples of colorectal carcinoma along with the corresponding clinical follow-up details were collected from the Department of Pathology, Nanfang Hospital over a period of more than 10 years. For determination of pan-Slit and Robo1 immunoreactivity [16], cytosolic staining of yellowish or brownish granules was graded as follows: 0 for background staining, 1 for faint staining, 2 for moderate staining and 3 for strong staining. In addition, positive staining areas in entire tissue section were graded as follows: 0 for $<10 \%, 1$ for $11-25 \%, 2$ for $26-50 \%$ and 3 for $51-$ $100 \%$. When combining these two parameters, $0-2$ and $\geq 3$ were defined as negative and positive for antigen, respectively.

\section{Statistical analysis}

The difference in frequency between patients with and without metastasis was evaluated using $\chi^{2}$-test. To compute correlation coefficients of immunostaining scores between two proteins, Spearman's rank correlation coefficient was used. Overall survival was represented in months, which was defined as the interval between the date of the surgery and the date of death or last follow-up. Overall survival curves were estimated by the Kaplan-Meier method, and the difference in survival was evaluated using the log-rank test. The $P$-values of $<0.05$ and 0.01 were considered statistically significant and very significant. All computations were made with R 2.9.0 (http://www.r-project.org).

\section{Acknowledgments}

We thank Biao Wang for preliminary studies and Michael J Franklin for editing the manuscript. We are grateful to Elaine Fuchs for the plasmid of the E-cadherin promoter luciferase reporter. This work was supported by grants from the Ministry of Science and Technology of China (2010CB529702 to XY and L-JW, 2010 CB529403 to Y-QD), National Science Foundation of China (30871304 to L-JW) and National Institutes of Health (CA126897, AI064743 to J-GG).

\section{References}

1 Kidd T, Brose K, Mitchell KJ, et al. Roundabout controls axon crossing of the CNS midline and defines a novel subfamily of evolutionarily conserved guidance receptors. Cell 1998; 92:205-215.

2 Brose K, Bland KS, Wang KH, et al. Slit proteins bind Robo receptors and have an evolutionarily conserved role in repulsive axon guidance. Cell 1999; 96:795-806.

3 Li HS, Chen JH, Wu W, et al. Vertebrate Slit, a secreted ligand for the transmembrane protein Roundabout, is a repellent for olfactory bulb axons. Cell 1999; 96:807-818.

4 Plump AS, Erskine L, Sabatier C, et al. Slit1 and Slit2 cooperate to prevent premature midline crossing of retinal axons in the mouse visual system. Neuron 2002; 33:219-232.

5 Bashaw GJ, Kidd T, Murray D, Pawson T, Goodman CS. Repulsive axon guidance: Abelson and Enabled play opposing roles downstream of the roundabout receptor. Cell 2000; 101:703-715.

6 Dickson BJ, Gilestro GF. Regulation of commissural axon path finding by Slit and its Robo receptors. Annu Rev Cell Dev Biol 2006; 22:651-675.

7 Wu JY, Feng LL, Park HT, et al. The neuronal repellent Slit inhibits leukocyte chemotaxis induced by chemotactic factors. Nature 2001; 410:948-952.

8 Guan $\mathrm{H}, \mathrm{Zu} \mathrm{G}$, Xie Y, et al. Neuronal repellent Slit2 inhibits dendritic cell migration and the development of immune responses. J Immunol 2003; 171:6519-26.

9 Kanellis J, Garcia GE, Li P, et al. Modulation of inflammation by Slit protein in vivo in experimental crescentic glomerulonephritis. Am J Pathol 2004; 165:341-352.

10 Prasad A, Qamri Z, Wu J, Ganju RK. Slit2/Robo1 modulates the CXCL12/CXCR4-induced chemotaxis of T cells. J Leukoc Biol 2007; 82:465-476.

11 Altay T, McLaughlin B, Wu JY, Park TS, Gidday JM. Slit modulates cerebrovascular inflammation and mediates neuroprotection against global cerebral ischemia. Exp Neurol 2007; 207:186-194.

12 Tole S, Mukovozov IM, Huang YW, et al. The axonal repellent, Slit2, inhibits directional migration of circulating neutrophils. J Leukoc Biol 2009; 86:1403-1415.

13 Schmid BC, Rezniczek G.A, Fabjani G, Yoneda T, Leodolter S, Zeillinger R. The neuronal guidance cue Slit2 induces targeted migration and may play a role in brain metastasis of breast cancer cells. Breast Cancer Res Treat 2007; 106:333-342.

14 Mertsch S, Schmitz N, Jeibmann A, Geng JG, Paulus W, Senner V. Slit2 involvement in glioma cell migration is mediated by Robol receptor. J Neurooncol 2008; 87:1-7.

15 Yuasa-Kawada J, Kinoshita-Kawada M, Rao Y, Wu JY. Deubiquitinating enzyme USP33/VDU1 is required for Slit signaling in inhibiting breast cancer cell migration. Proc Natl Acad Sci USA 2009; 106:14530-14535.

16 Wang B, Xiao Y, Ding, B, et al. Induction of tumor angiogenesis by Slit-Robo signaling and inhibition of cancer growth by blocking Robo activity. Cancer Cell 2003; 4:19-29.

17 Wang LJ, Zhao Y, Han B, et al. Targeting Slit-Roundabout signaling inhibits tumor angiogenesis in chemical-induced squamous cell carcinogenesis. Cancer Sci 2008; 99:510-517.

18 Urbich C, Rössig L, Kaluza D, et al. HDAC5 is a repressor of angiogenesis and determines the angiogenic gene expression pattern of endothelial cells. Blood 2009; 113:5669-5679.

19 Shen F, Liu X, Geng JG, Guo SW. Increased immunoreac- 
tivity to Slit/Robol in ovarian endometriomas. Am J Pathol 2009; 175:479-488.

20 Zhang B, Dietrich UM, Geng JG, Bicknell R, Esko JD, Wang L. Repulsive axon guidance molecule Slit3 is a novel angiogenic factor. Blood 2009; 114:4300-4309.

21 Ma S, Liu X, Geng JG, Guo SW. Increased SLIT immunoreactivity as a biomarker for recurrence in endometrial carcinoma. Am J Obstet Gynecol 2010; 202:68.e1-68.e11.

22 Yang XM, Han HX, Sui F, Dai YM, Chen M, Geng JG. SlitRobo signaling mediates lymphangiogenesis and promotes tumor lymphatic metastasis. Biochem Biophys Res Commun 2010; 396:571-577.

23 Grunert S, Jechlinger M, Beug H. Diverse cellular and molecular mechanism contribute to epithelial plasticity and metastasis. Nat Rev Mol Cell Biol 2003; 4:657-665.

24 Gumbiner BM. Regulation of cadherin-mediated adhesion in morphogenesis. Nat Rev Mol Cell Biol 2005; 6:622-634.

25 Radisky DC. Epithelial-mesenchymal transition. J Cell Sci 2005; 118:4325-4326.

26 Thiery JP, Sleeman JP. Complex networks orchestrate epithelial-mesenchymal transitions. Nat Rev Mol Cell Biol 2006; 7:131-142.

27 Pokutta S, Weis WI. Structure and mechanism of cadherin and catenins in cell-cell contacts. Annu Rev Cell Dev Biol 2007; 23:237-261.

28 Maina F, Pante G, Helmbacher F, et al. Coupling Met to specific pathways results in distinct developmental outcomes. Mol Cell 2001; 7:1293-1306.

29 Lu Z, Ghosh S, Wang Z, Hunter T. Downregulation of caveolin-1 function by EGF leads to the loss of E-cadherin, increased transcriptional activity of $\beta$-catenin, and enhanced tumor cell invasion. Cancer Cell 2003; 4:499-515.

30 Shimizu Y, Yamamichi N, Saitoh K, et al. Kinetics of V-srcinduced epithelial-mesenchymal transition in developing glandular stomach. Oncogene 2003; 22:884-893.

31 Palacios F, Tushir JS, Fujita Y, D’Souza-Schorey C. Lysosomal targeting of E-cadherin: a unique mechanism for the downregulation cell-cell adhesion during epithelial to mesenchymal transitions. Mol Cell Biol 2005; 25:389-402.

32 Janda E, Nevolo M, Lehmann K, Downward J, Beng H, Grieco M. Raf plus TGF- $\beta$-dependent EMT is initiated by endocytosis and lysosomal degradation of E-cadherin. Oncogene 2006; 25:7117-7130.

33 Bryant DM, Kerr MC, Hammond LA, et al. EGF induces macropinocytosis and SNX1-modulated recycling of E-cadherin. J Cell Sci 2007; 120:1818-1828.

34 Wheelock MJ, Shintani Y, Maeda M, Fukumoto Y, Johnson KR. Cadherin switching. J Cell Sci 2008; 121:727-735.

35 Shen Y, Hirsch DS, Sasiela CA, Wu WJ. Cdc42 regulates Ecadherin ubiquitination and degradation through an epidermal growth factor receptor to Src-mediated pathway. $J$ Biol Chem 2008; 283:5127-5137.

36 Kobielak A, Fuchs E. $\alpha$-catenin: at the junction of intercellular adhesion and actin dynamics. Nat Rev Mol Cell Biol 2004; 5:614-625.

37 Gröne J, Doebler O, Loddenkemper C, Hotz B, Buhr HJ, Bhargava S. Robo1/Robo4: differential expression of angiogenic markers in colorectal cancer. Oncol Rep 2006; 15:14371443.
38 Labbe E, Lock L, Letamendia A, et al. Transcriptional cooperation between the transforming growth factor and Wnt pathways in mammary and Intestinal tumorigenesis. Cancer Res 2007; 67:75-84.

39 Fujita Y, Krause G, Scheffner M, et al. Hakai, a c-Cbl-like protein, ubiquitinates and induces endocytosis of the E-cadherin complex. Nat Cell Biol 2002; 4:222-231.

40 Qian L, Liu J, Bodmer R. Slit and Robo control cardiac cell polarity and morphogenesis. Curr Biol 2005; 15:2271-2278.

41 MacMullin A, Jacobs JR. Slit coordinates cardiac morphogenesis in Drosophila. Dev Biol 2006; 293:154-164.

42 Santiago-Martinez E, Soplop NH, Kramer SG. Lateral positioning at the dorsal midline: Slit and Roundabout receptors guide Drosophila heart cell migration. Proc Natl Acad Sci USA 2006; 103:12441-12446.

43 Santiago-Martinez E, Soplop N.H, Kramer SG. Repulsion by Slit and Roundabout prevents Shotgun/E-cadherin-mediated cell adhesion during Drosophila heart tube lumen formation. J Cell Biol 2008; 182:241-248.

44 Medioni C, Astier M, Zmojdzian M, Jagla K, Semeriva M. Genetic control of cell morphogenesis during Drosophila melanogaster cardiac tube formation. J Cell Biol 2008; 182:249261.

45 Dallol A, Da Silva NF, Viacava P, et al. SLIT2, a human homolog of the Drosophila Slit2 gene, has tumor suppressor activity and is frequently inactivated in lung and breast cancers. Cancer Res 2002; 62:5874-5880.

46 Dallol A, Krex D, Hesson L, Eng C, Maher ER, Latif F. Frequent epigenetic inactivation of the SLIT2 gene in gliomas. Oncogene 2003; 22:4611-4616.

47 Dallol A, Morton D, Maher ER, Latif F. SLIT2 axon guidance molecule is frequently inactivated in colorectal cancer and suppresses growth of colorectal carcinoma cells. Cancer Res 2003; 63:1054-1058.

48 Sharma G, Mirza S, Prasad CP, Srivastava A, Gupta SD, Ralhan R. Promoter hypermethylation of p16INK4A, p14ARF, cyclin D2 and Slit2 in serum and tumor DNA from breast cancer patients. Life Sci 2007; 80:1873-1881.

49 Singh RK, Indra D, Mitra S, et al. Deletions in chromosome 4 differentially associated with the development of cervical cancer: evidence of Slit2 as a candidate tumor suppressor gene. Hum Genet 2007; 122:71-81.

50 Dunwell TL, Dickinson RE, Stankovic T, et al. Frequent epigenetic inactivation of the SLIT2 gene in chronic and acute lymphocytic leukemia. Epigenetics 2009; 4:265-269.

51 Jin J, You H, Yu B, et al. Epigenetic inactivation of SLIT2 in human hepatocellular carcinomas. Biochem Biophys Res Commun 2009; 379:86-91.

52 Prasad A, Paruchuri V, Preet A, Latif F, Ganju RK. Slit-2 induces a tumor-suppressive effect by regulating beta-catenin in breast cancer cells. J Biol Chem 2008; 283:26624-26633.

53 Tseng RC, Lee SH, Hsu HS, et al. SLIT2 attenuation during lung cancer progression deregulates beta-catenin and E-cadherin and associates with poor prognosis. Cancer Res 2010; 70:543-551.

54 Astuti D, Da Silva NF, Dallol A, et al. SLIT2 promoter methylation analysis in neuroblastoma, Wilms' tumour and renal cell carcinoma. Br J Cancer 2004; 90:515-521.

55 Dammann R, Strunnikova M, Schagdarsurengin U, et al. CpG 
island methylation and expression of tumour-associated genes in lung carcinoma. Eur J Cancer 2005; 41:1223-1236.

56 Dietrich D, Lesche R, Tetzner R, et al. Analysis of DNA methylation of multiple genes in microdissected cells from formalin-fixed and paraffin-embedded tissues. $J$ Histochem Cytochem 2009; 57:477-489.

57 Narayan G, Goparaju C, Arias-Pulido H, et al. Promoter hypermethylation-mediated inactivation of multiple Slit-Robo pathway genes in cervical cancer progression. Mol Cancer 2006; 5:16-25.

58 Li L, Backer J, Wong AS, Schwanke EL, Stewart BG, Pasdar M. Bcl-2 expression decreases cadherin-mediated cell-cell adhesion. J Cell Sci 2003; 116:3687-3700.

59 Planas-Silva MD, Waltz PK. Estrogen promotes reversible epithelial-to-mesenchymal-like transition and collective motility in MCF-7 breast cancer cells. J Steroid Biochem Mol Biol 2007; 104:11-21.

60 Laffin B, Wellberg E, Kwak HI, et al. Loss of singleminded$2 \mathrm{~s}$ in the mouse mammary gland induces an epithelial-mesenchymal transition associated with up-regulation of slug and matrix metalloprotease 2. Mol Cell Biol 2007; 28:1936-1946.

61 Arima Y, Inoue Y, Shibata T, et al. Rb depletion results in deregulation of E-cadherin and induction of cellular phenotypic changes that are characteristic of the epithelial-to-mesenchymal transition. Cancer Res 2008; 68:5104-5112.

62 Rhee J, Mahfooz NS, Arregui C, Lilien J, Balsamo J, VanBerkum MF. Activation of the repulsive receptor Roundabout inhibits N-cadherin mediated cell adhesion. Nat Cell Biol 2002; 4:798-805.

63 Rhee J, Buchan T, Zukerberg L, Lilien J, Balsamo J. Cables links Robo-bound Abl kinase to $\mathrm{N}$-cadherin bound $\beta$-catenin to mediate Slit-induced modulation of adhesion and transcription. Nat Cell Biol 2007 9:883-892.

64 Wong K, Park HT, Wu JY, Rao Y. Slit proteins: molecular guidance cues for cells ranging from neurons to leukocytes. Curr Opin Genet Dev 2002; 12:583-591.

65 Wang B, Geng JG. Highly-conserved mechanisms of cell migration and contact in immune and nervous systems. Med Hypotheses Res 2005; 2:315-323.

66 Heldin CH, Lanström M, Moustakas A. Mechanism of TGF- $\beta$ signaling to growth arrest, apoptosis, and epithelial-mesenchymal transition. Curr Opin Cell Biol 2009; 21:166-176.

67 Gressner OA, Rizk MS, Kovalenko E, Weiskirchen R, Gressner AM. Changing the pathogenetic roadmap of liver fibrosis? Where did it start; where will it go? J Gastroenterol Hepatol 2008; 23:1024-1035.
68 Battaglia S, Benzoubir N, Nobilet S, et al. Liver cancer-derived hepatitis $\mathrm{C}$ virus core proteins shift TGF-beta responses from tumor suppression to epithelial-mesenchymal transition. PLoS One 2009; 4:e4355.

69 Gregorieff A, Clevers H. Wnt signaling in the intestinal epithelium: from endoderm to cancer. Genes Dev 2005; 19:877890 .

70 Heuberger J, Birchmeier W. Interplay of cadherin-mediated cell adhesion and canonical Wnt signaling. Cold Spring Harb Perspect Biol 2010; 2:a002915.

71 Gregory PA, Bracken CP, Goodall GJ. MicroRNAs as regulators of epithelial-mesenchymal transition. Cell Cycle 2008; 7:3112-3118.

72 Fritz JL, VanBerkum MF. Calmodulin and Son of sevenless dependent signaling pathways regulate midline crossing of axon in the Drosophila CNS. Development 2000; 127:19912000.

73 Wong K, Ren XR, Huang YZ, et al. Signal transduction in neuronal migration: roles of GTPase activating proteins and the small GTPase cdc42 in the Slit-Robo pathway. Cell 2001; 107:209-221.

74 Huminiecki L, Gorn M, Suchting S, Poulsom R, Bicknell R. Magic roundabout is a new member of the roundabout receptor family that is endothelial specific and expressed at sites of active angiogenesis. Genomics 2002; 79:547-552.

75 Bedell VM, Yeo SY, Park KW, et al. Roundabout4 is essential for angiogenesis in vivo. Proc Natl Acad Sci USA 2005; 102:6373-6378.

76 Jones CA, London NR, Chen H, et al. Robo4 stabilizes the vascular network by inhibiting pathologic angiogenesis and endothelial hyperpermeability. Nat Med 2008; 14:448-453.

77 Sheldon H, Andre M, Legg JA, et al. Active involvement of Robo1 and Robo4 in filopodia formation and endothelial cell motility mediated via WASP and other actin nucleationpromoting factors. FASEB J 2009; 23:513-522.

78 Jones CA, Nishiya, N, London NR, et al. Slit2-Robo4 signalling promotes vascular stability by blocking Arf6 activity. Nat Cell Biol 2009; 11:1325-1331.

79 Wang HB, Wang JT, Zhang L, et al. P-selectin primes leukocyte integrin activation during inflammation. Nat Immunol 2007; 8:882-892.

80 Xia YF, Ye BQ, Li YD, et al. Andrographolide attenuates inflammation by inhibition of NF-kappa B activation through covalent modification of reduced cysteine 62 of p50. J Immunol 2004; 173:4207-4217.

(Supplementary information is linked to the online version of the paper on the Cell Research website.) 